\title{
Structural parameters for globular clusters in M31 and generalizations for the fundamental plane ${ }^{1}$
}

\author{
Pauline Barmby \\ Harvard-Smithsonian Center for Astrophysics, 60 Garden St., Mailstop 65, Cambridge, \\ MA 02138 \\ Dean E. McLaughlin \\ Department of Physics and Astronomy, University of Leicester, University Road, Leicester \\ LE1 7RH, UK \\ William E. Harris \\ Department of Physics and Astronomy, McMaster University, Hamilton, ON L8S 4M1, \\ Canada \\ Gretchen L.H. Harris \\ Department of Physics and Astronomy, University of Waterloo, Waterloo, ON N2L 3G1, \\ Canada \\ and \\ Duncan A. Forbes \\ Centre for Astrophysics and Supercomputing, Swinburne University, Hawthorn, VIC 3122, \\ Australia
}

\begin{abstract}
The structures of globular clusters (GCs) reflect their dynamical states and past histories. High-resolution imaging allows the exploration of morphologies of clusters in other galaxies. Surface brightness profiles from new Hubble Space Telescope observations of 34 globular clusters in M31 are presented, together with fits of several different structural models to each cluster. M31 clusters appear to be adequately fit by standard King models, and do not obviously require alternate descriptions with relatively stronger halos, such as are needed to fit many GCs in other nearby galaxies. The derived structural parameters are combined with corrected versions of those measured in an earlier survey to
\end{abstract}


construct a comprehensive catalog of structural and dynamical parameters for M31 GCs with a sample size similar to that for the Milky Way. Clusters in M31, the Milky Way, Magellanic Clouds, Fornax dwarf spheroidal and NGC 5128 define a very tight fundamental plane with identical slopes. The combined evidence for these widely different galaxies strongly reinforces the view that old globular clusters have near-universal structural properties regardless of host environment.

Subject headings: galaxies: star clusters — galaxies: individual (M31) — globular clusters: general

\section{Introduction}

Globular clusters (GCs) have long been recognized as unique dynamical laboratories and unique markers of galaxy history. The integrated properties of GCs, such as age and metallicity, are believed to reflect conditions in the early stages of galaxy formation (Brodie \& Strader 2006). The spatial structure and kinematics of GCs reflect both their formation conditions and dynamical evolution within the tidal fields of their host galaxies.

Structures of GCs as described by surface brightness or velocity profiles can be fit by a number of different models. These models can be used to compute numerous parameters describing various aspects of cluster structure, but four independent parameters suffice to completely define a single-mass model. It is helpful to think of cluster structures as being defined by spatial scale, spatial concentration, total luminosity, and a relation between luminosity and mass, but many different parameter choices are possible. Djorgovski (1995) and McLaughlin (2000) used formally different, but entirely equivalent, sets of structural parameters to show that globular clusters in the Milky Way do not occupy the full four-dimensional parameter space but instead define a remarkably narrow 'fundamental plane'. The same appears to be true for clusters in M31 (Diorgovski et al. 1997; Dubath \& Grillmair 1997; Barmby et al. 2002), in NGC 5128 (Harris et al. 2002), and in M33 (Larsen et al. 2002). This evidence indicates that the formation and evolution processes for normal globular clusters were similar in a wide variety of environments (Harris 2003).

\footnotetext{
${ }^{1}$ Based on observations made with the NASA/ESA Hubble Space Telescope, obtained at the Space Telescope Science Institute, which is operated by the Association of Universities for Research in Astronomy, Inc., under NASA contract NAS 5-26555. These observations are associated with programs \#8664, \#9719, \# 9767 and \#10260.
} 
The globular cluster fundamental plane is similar in character, but different in detail, to that of elliptical galaxies (for an introduction, see Burstein et al. 1997). There have been several suggestions that at least some of the most massive GCs in the Milky Way and M31 are in fact the nuclei of destroyed dwarf galaxies (e.g. Tsuchiya et al. 2003; Hilker \& Richtler 2000), or that they are related to ultra-compact dwarf galaxies (Hasegan et al. 2005). Both dwarf galaxy nuclei and UCDs occupy somewhat different regions of the fundamental plane than do GCs, but structural properties have been measured for only a few objects in this transition mass region between globulars and galaxies. Combining samples of clusters from a number of nearby galaxies - where structures can be accurately measured with highresolution imaging from the Hubble Space Telescope (HST) - is a way to improve the sample statistics particularly for the upper end of the GC mass range.

To further understand the globular cluster fundamental plane, we have carried out a survey of massive globulars in M31 and NGC 5128 using the HST Advanced Camera for Surveys (ACS; Ford et al. 2002). Other papers from this program discuss the NGC 5128 cluster sample (Harris et al. 2006), the techniques for measurement of those clusters' structural parameters (McLaughlin et al. 2007a), and the comparison between massive globulars, nuclear star clusters, and 'dwarf-globular transition objects' (McLaughlin et al. 2007b). This paper presents the new M31 observations and structural parameters, compiles a publicly-available catalog of measurements of structural parameters for the largest-ever sample of M31 clusters, and compares the resulting 'fundamental planes' of globular cluster parameters in different galaxies. Throughout this paper we assume a distance to M31 of $780 \mathrm{kpc}$ (1 pc subtends $0 \prime 26$ ) and use the cluster nomenclature of the Revised Bologna Catalogue (Galleti et al. 2006).

\section{Observational material}

We have used material from several different HST programs. Program GO-10260 (PI W. Harris) obtained ACS Wide Field Channel (WFC) images centered on six clusters in M31 and twelve clusters in NGC 5128, selected to be those having $M_{V}<-10.5$ and (for M31) no previous HST observations. Exposure times were $2370 \mathrm{~s}$ (one orbit split into three dither positions) in both the F606W and F814W filters. Most of the GO-10260 M31 observations were made in the period 2004 September 29-2004 October 01. Program GO-9719 (PI T. Bridges) obtained ACS High Resolution Channel (HRC) images of three M31 clusters using exposure times of $2020 \mathrm{~s}$ in F606W and $2680 \mathrm{~s}$ in F814W in 2003 August and September. Program GO-8664 (PI W. Harris) targeted 24 M31 clusters with a range of properties (as well as NGC 5128 clusters, discussed in Harris et al. 2002) for imaging with STIS in snapshot 
mode; exposure times were $480 \mathrm{~s}$ in the unfiltered 50CCD/CL imaging mode. These data were obtained in fall 2000 and winter 2001. Of the 24 STIS targets, one (B329) is clearly a galaxy, and two more (B391 and B340) had faulty coordinates and were not on the images. Cluster B396 was bisected by the edge of the STIS image but the data were still usable.

The cluster G001 (Mayall II) is one of the most massive in M31 and has been the subject of numerous recent investigations. It is often likened to the Milky Way's own massive cluster $\omega$ Cen; both clusters have been suggested as possible remnant dwarf galaxy cores (Meylan et al. 2001). There has been some discussion about whether G001 contains a central black hole (Gebhardt et al. 2002; Baumgardt et al. 2003; Gebhardt et al. 2005) and also some widely differing measurements of its structural parameters (see discussion in Barmby et al. 2002). Gebhardt et al. (2005) used new HST/ACS observations of G001 (obtained as part of GO-9767) to produce a surface brightness profile as part of an effort to constrain the mass of any putative central black hole, but those authors did not give values for the cluster's structural parameters. Ma et al. (2007) fit King (1966) models to the ACS data. Because of the importance of G001, we decided to re-analyze the Gebhardt et al. (2005) observations, which consist of six exposures totaling 41 minutes of integration time with the ACS HRC in the F555W filter.

The HST images offered the possibility of detecting new clusters or confirming additional cluster candidates other than the targets. All the M31 images were searched for additional clusters and inspected at the positions of cataloged cluster candidates. A number of additional cluster candidates appeared in the ACS images: B041, B056D, B061, B081D, B088D, B090, B102, B147, B162 and M027. We also (independently) re-discovered the new cluster B515 reported by Galleti et al. (2006). All of these candidates appear to be clusters except for B102, which is apparently a star.2 Interestingly, B147, which had been classified as a star based on velocity dispersion measurements by Dubath \& Grillmair (1997), is clearly a resolved cluster (see Figure 1). This object is also associated with an X-ray source (Trudolyubov \& Priedhorsky 2004). Figure 1 shows images of B147 and the highly elliptical cluster B088 demonstrating the power of HST imaging for resolving M31 globular clusters.

To generate the largest possible sample of M31 globulars for fundamental plane analysis, we also include most of the clusters with structural parameters measured from images taken with HST's Wide Field and Planetary Camera 2 (WPFC2), reported in Barmby et al. (2002). The clusters from Barmby et al. (2002) included in the present sample include 59 objects with HST/WFPC2 photometry in the $V$-band and good measurements of $r_{0}$ and

\footnotetext{
${ }_{2}^{2}$ Galleti et al. (2006) independently came to the same conclusions about the nature of B056D, B102, and B162.
} 
c. The central surface brightness values for these clusters have had an important error corrected. As also discussed in McLaughlin et al. (2007a), the software used to fit structural models in Barmby et al. (2002) and also Harris et al. (2002) did not return a model central surface brightness value; instead both papers reported $\mu_{V, 0}$ as the surface brightness of the central pixel of the PSF-convolved model image. As such, the values reported in Barmby et al. (2002) are systematically too faint. We re-computed $\mu_{V, 0}$ from the King (1966) $c$ and $r_{0}$ values reported in Barmbv et al. (2002) and the integrated $V$ magnitudes reported in Barmby et al. (2000) or Barmby \& Huchra (2001). The median surface brightness change is $\Delta \mu_{V, 0}=-0.33 \mathrm{mag}$, with a large scatter. The maximum change is about $1.5 \mathrm{mag}$, and there were several clusters, both bright and faint, whose central surface brightnesses were nearly unchanged in the re-calculation. Using the model-fitting software of both McLaughlin et al. (2007a) and Barmby et al. (2002) on a small number of M31 clusters showed that both recovered the the same $r_{0}$ and $c$ values to within the uncertainties, so the two sets of measurements should be consistent.

To summarize, the new observations include 15 clusters observed using the ACS in either the Wide Field or High-Resolution Channel, in both F606W and F814W filters; and 19 clusters observed in the unfiltered mode using STIS. The observations of cluster G001 with ACS/HRC in the F555W filter are not new but are re-analyzed here. There are therefore 50 surface-brightness profiles for 34 distinct objects (one of the STIS clusters, B023, is also in the ACS sample and has thus been observed three times). Adding the 59 objects from Barmby et al. (2002), the sample of M31 clusters totals 93 objects, with some later omitted from the fundamental plane analyses (see $\S 3.3$ ). Estimates of the total size of the M31 GCS vary: one recent value is $N_{g c}=460 \pm 70$ (Barmby \& Huchra 2001). Thus the HST sample comprises only a fraction of the total M31 population, but it is comparable in size to the full sample of non-core-collapsed Milky Way clusters considered by McLaughlin (2000), and about 10\% larger than the sample of 85 Milky Way clusters analyzed by McLaughlin \& van der Marel (2005). We have not attempted to generate a complete sample of clusters observed with HST by searching the Archive for clusters serendipitously observed in data taken after the Barmby \& Huchra (2001) sample was compiled; however we expect that the present sample should be representative of M31 GCs particularly at the high-luminosity end. The exception is low-surface-brightness clusters for which our present analysis method is unsuitable: these include both faint clusters, some discussed in the following section, and also the 'extended luminous' clusters discovered by Huxor et al. (2005) (see also §6.3). 


\section{Data analysis}

The data analysis procedures used for these data are described in detail by McLaughlin et al. (2007a). Here the procedures are briefly summarized, and some details specific to the M31 observations noted.

\subsection{Surface Brightness Profiles}

The STScI-pipeline output drizzled images were used to measure cluster shapes with ELLIPSE in IRAF. Cluster center positions were fixed at values derived by centroiding and elliptical isophotes were fit to the data, with no sigma-clipping. A first pass of ELLIPSE was run in the usual way, with ellipticity and position angle allowed to vary with the isophote semi-major axis. Luminosity-weighted averages of ellipticity and position angle were determined from the results. In the second pass of ELLIPSE, surface brightness profiles on fixed, zero-ellipticity isophotes were measured (this was required because we chose to fit circular models for both the intrinsic cluster structure and the PSF). For several low-density clusters (B290 and B423 observed with STIS; B081D, B088D, B515, and M027 observed with ACS), a sensible, monotonically-decreasing profile could not be obtained: no models were fit to these clusters. Table 1 reports the average ellipticities, position angles, and aperture magnitudes measured from the ACS and STIS images, and tabulates some additional reference data (metallicity, reddening, galactocentric distance) for all of the M31 clusters. For 11 of the clusters, there are no metallicity estimates in the literature, so we have simply assigned $[\mathrm{Fe} / \mathrm{H}]=-1.2 \pm 0.6$ to all of them (the mean and standard deviation of the metallicity distribution of the M31 GC system; Barmby et al. 2000).

The raw output from ELLIPSE is in units of cts/s per pixel, which is converted to cts/s per square arcsecond by multiplying by $400=\left(1 \mathrm{px} / 0^{\prime \prime} .05\right)^{2}$ for $\mathrm{WFC}, 1600=\left(1 \mathrm{px} / 0^{\prime \prime} .025\right)^{2}$ for $\mathrm{HRC}$, or $387.5=\left(1 \mathrm{px} / 0^{\prime \prime} 0508\right)^{2}$ for STIS. To transform the ACS counts to surface brightness calibrated on the VEGAMAG system (from the ACS Handbook)

$$
\mu / \mathrm{mag} \operatorname{arcsec}^{-2}=z-2.5 \log \left(\operatorname{cts} / \mathrm{s} / \square^{\prime \prime}\right)
$$

where the zeropoints are $z=(25.255,26.398,25.893,25.501,24.849)$ for HRC/F555W, WFC/F606W, $\mathrm{HRC} / \mathrm{F} 606 \mathrm{~W}, \mathrm{WFC} / \mathrm{F} 814 \mathrm{~W}$, and $\mathrm{HRC} / \mathrm{F} 814 \mathrm{~W}$ respectively. The STIS data were taken in unfiltered ("clear" or CL) mode, for which Harris et al. (2002) derive

$$
\mu_{V} / \mathrm{mag} \operatorname{arcsec}^{-2}=(26.29 \pm 0.05)-2.5 \log \left(\operatorname{cts} / \mathrm{s} / \square^{\prime \prime}\right)
$$

in the standard $V$ bandpass. 
We allow for the occasional over-subtraction of sky in the automatic reduction pipeline, and hence "negative" counts in some pixels, by working in terms of linear intensity instead of surface brightness in magnitudes. Given $3 M_{\odot, F 606 W}=4.64, M_{\odot, F 814 W}=4.14$, and $M_{\odot, V / F 555 W}=4.83$, the calibration above yields

$$
\begin{aligned}
& I_{F 555 W} / L_{\odot} \mathrm{pc}^{-2} \simeq 2.8766 \times\left(\mathrm{cts} / \mathrm{s} / \square^{\prime \prime}\right) \quad[\mathrm{HRC}] \\
& I_{F 606 \mathrm{~W}} / L_{\odot} \mathrm{pc}^{-2} \simeq 0.8427 \times\left(\mathrm{cts} / \mathrm{s} / \square^{\prime \prime}\right) \quad[\mathrm{WFC}] \\
& I_{F 606 \mathrm{~W}} / L_{\odot} \mathrm{pc}^{-2} \simeq 1.3418 \times\left(\mathrm{cts} / \mathrm{s} / \square^{\prime \prime}\right) \quad[\mathrm{HRC}] \\
& I_{F 814 W} / L_{\odot} \mathrm{pc}^{-2} \simeq 1.2147 \times\left(\mathrm{cts} / \mathrm{s} / \square^{\prime \prime}\right) \quad[\mathrm{WFC}] \\
& I_{F 814 W} / L_{\odot} \mathrm{pc}^{-2} \simeq 2.2145 \times\left(\mathrm{cts} / \mathrm{s} / \square^{\prime \prime}\right) \quad[\mathrm{HRC}] \\
& I_{V} / L_{\odot} \mathrm{pc}^{-2} \simeq 1.1089 \times\left(\mathrm{cts} / \mathrm{s} / \square^{\prime \prime}\right) \quad[\mathrm{STIS}] .
\end{aligned}
$$

Converting from luminosity density in $L_{\odot} \mathrm{pc}^{-2}$ to surface brightness is done according to $\mu_{814} / \mathrm{mag} \operatorname{arcsec}^{-2}=25.712-2.5 \log \left(I_{814} / L_{\odot} \mathrm{pc}^{-2}\right)$ for $\mathrm{F} 814 \mathrm{~W} ; \mu_{606} / \mathrm{mag} \operatorname{arcsec}^{-2}=$ $26.212-2.5 \log \left(I_{606} / L_{\odot} \mathrm{pc}^{-2}\right)$ for $\mathrm{F} 606 \mathrm{~W} ; \mu_{V, 555} / \mathrm{mag} \operatorname{arcsec}^{-2}=26.402-2.5 \log \left(I_{V, 555} / L_{\odot} \mathrm{pc}^{-2}\right)$ for standard $V$ and $\mathrm{F} 555 \mathrm{~W}$.

The ELLIPSE profiles extended to $R=12$ ' $8 \simeq 48$ pc from all images of the sample clusters, and thus in the model fitting a constant-background term is included to allow for any errors in sky subtraction in the automatic pipeline. Each profile was inspected, and one or two very discrepant points in each of a handful of clusters were excluded by hand. Inspection of the data quality images for the WFC images showed that, due to an unfortunate choice of CCD gain, the cores of several bright clusters (B042, B063, B082, B147, and B151) were saturated, with raw intensities above a nominal limit of $70 \mathrm{cts} \mathrm{s}^{-1} \mathrm{pix}^{-1}$ $\left(28,000 \mathrm{cts} \mathrm{s}^{-1} \operatorname{arcsec}^{-2}\right.$, or $\left.I_{F 606 W} \simeq 23,600 L_{\odot} \mathrm{pc}^{-2}\right)$ all the way out to $R \sim 2^{\prime \prime}=7.5 \mathrm{pc}$ (or $\sim 40$ pix) in most cases. This is well beyond the FWHM of the WFC PSF in either F606W or F814W; see below. Intensities as high as this were not included in the model fitting, and thus the core parameters of these five clusters are constrained only very indirectly. None of the HRC or STIS images is saturated.

Table 2 gives the final, calibrated intensity profiles for all clusters. These are not corrected for extinction, which is discussed below. The reported F606W- and F814W-band intensities are calibrated on the VEGAMAG scale, while the STIS data are on the standard $V$ system. The final column gives a flag for every point, which can take one of four values. "BAD" indicates that the intensity value is deemed dubious because it strongly deviates from its neighbors or is obviously affected by nearby bright stars or image artifacts. "SAT" indicates that the isophotal intensity is above the imposed saturation limit of $70 \mathrm{cts} \mathrm{s}^{-1}$ pix $^{-1}$

${ }^{3}$ http://www.ucolick.org/ cnaw/sun.html 
"DEP" indicates that the radius is inside $R<2$ pix $=0$ "' 1 and the isophotal intensity is dependent on its neighbors. The ELLIPSE output includes brightnesses for 15 radii inside 2 pixels, but these are all measured from the same 13 central pixels and are clearly not statistically independent. To avoid excessive weighting of the central regions of the cluster in the fits, only intensities at radii $R_{\min }, R_{\min }+(0.5,1.0,2.0 \mathrm{px}), R>2.5 \mathrm{px}$, were used. "OK" indicates that none of the above apply and the point is used in model fitting.

\subsection{Point-Spread Functions}

M31 clusters are clearly resolved with HST, but their observed core structures are still affected by the PSF. We chose not to deconvolve the data, instead convolving the structural models with a simple analytic description of the PSF before fitting.

To estimate the PSF for the WFC, ELLIPSE was used (again with circular symmetry enforced) to produce intensity profiles out to radii of about $2^{\prime \prime}$ (40 pixels) for a number of isolated stars on a number of images, and combined them to produce a single, average PSF. This was done separately for the F606W and F814W filters. We originally tried to fit these with simple Moffat profiles (with backgrounds added), but found that a better description was given by a function of the form

$$
I_{\mathrm{PSF}} / I_{0}=\left[1+\left(R / r_{0}\right)^{3}\right]^{-\beta / 3}
$$

The derived $r_{0}$ and $\beta$ values, and the implied FWHMs, are listed in Table 3. Although the WFC PSF is known to vary over the instrument's field of view, McLaughlin et al. (2007a) show that the model-fitting results are insensitive to this effect.

For HRC data, the same functional form (eq.4) was fitted to the PSF images in F555W, F606W and F814W given by Anderson \& King (2005) with the results also listed in Table 3. In the case of STIS, a similar procedure was followed (see Harris et al. 2002) and it was found that a standard Moffat function, $I_{\mathrm{PSF}} \propto\left[1+\left(R / r_{0}\right)^{2}\right]^{-\beta / 2}$, gives an adequate fit to the PSF, with parameters also listed in Table 3. These are the functions convolved with the structural models before fitting to the observed intensity profiles.

\subsection{Extinction and Color Corrections}

The effective wavelengths of the ACS F606W and F814W filters are $\lambda_{\text {eff }}=5918 \AA$ and $\lambda_{\text {eff }}=8060 \AA$ (Sirianni et al. 2005), so that from Cardelli et al. (1989) $A_{606} \simeq 2.8 \times E(B-V)$ and $A_{814} \simeq 1.8 \times E(B-V)$-both of which are also found by Sirianni et al. (2005). This 
implies, rather conveniently, that $E(F 606 W-F 814 W) \simeq E(B-V)$. Individual reddening estimates - the sum of foreground and M31-internal reddening - are available for most of the clusters in the sample, derived using the procedure described by Barmby et al. (2000). The Burstein \& Heiles (1984) foreground value of $E(B-V)=0.08$ mag is adopted for clusters without individual measurements.

Sirianni et al. (2005) give transformations from ACS F606W and F814W magnitudes to standard $V$ and $I$ for both WFC and HRC measurements, including linear and quadratic dependences on de-reddened $(V-I)_{0}$ color (their Tables 22 and 23). However, measured $(V-I)$ is not available for 5 of the 15 clusters in the present ACS sample, and thus we chose instead to measure aperture $(F 606 W-F 814 W)$ colors from the current data, and manipulate the Sirianni et al. relations to find $(V-F 606 W)$ and $(V-F 814 W)$ indices.

Comparing the various "observed" and "synthetic" transformations from intrinsic $F 606 W$ magnitudes and $(V-I)_{0}$ colors to $V$ mags in Sirianni et al. (2005), it quickly becomes clear that there are systematic differences between them, at the level of $\sim 0.07$ mag in the color range $0.8 \lesssim(V-I)_{0} \lesssim 1.2$ appropriate for old clusters with sub-solar metallicities. However, a simple linear relation,

$$
(V-F 606 W)_{0}=-0.038+0.236(V-I)_{0}
$$

both gives a good estimate of the average of the Sirianni et al. relations (for VEGAMAG F606W magnitudes) and agrees very well with the predictions for old clusters in the populationsynthesis models of Maraston (1998, 2005). [VEGAMAG $(V-F 606 W)_{0}$ and $(V-F 814 W)_{0}$ indices were kindly computed for us, as functions of cluster age and metallicity, by C. Maraston.]

To go further, VEGAMAG $F 814 W$ magnitudes must be related to $I$. All of the WFC and HRC transformations in Sirianni et al. (2005) imply that $(F 814 W-I)_{0} \approx 0$ to within $\pm 0.01 \mathrm{mag}$ or so, while the population-synthesis models of Maraston have a roughly constant $(F 814 W-I) \simeq 0.04$ for $0.8 \lesssim(V-I)_{0} \lesssim 1.2$. Substituting $(F 814 W-0.04)$ for $I$ in equation (5) yields

$$
(V-F 606 W)_{0}=-0.04+0.31(F 606 W-F 814 W)_{0}
$$

and then trivially,

$$
(V-F 814 W)_{0}=-0.04+1.31(F 606 W-F 814 W)_{0},
$$

both with estimated rms scatter of about \pm 0.05 mag. These conversions were applied to both WFC and HRC data, after correcting the calibrated $F 606 W$ and $F 814 W$ magnitudes/intensities for extinction. 
Calibrating the other observations onto the standard $V$ scale was more straightforward. The ACS/HRC F555W filter is similar to the standard $V$ filter, and the data for G001 were calibrated using the extinction ratio and transformation given by Sirianni et al. (2005) (their Tables 15 and 23). The data for the 19 STIS clusters are already calibrated on the standard $V$ scale Harris et al. (2002), as are the WFPC2 data from Barmby \& Huchra (2001). The reddening values used to correct for extinction are recorded in Table 1.

Given the color transformations above, the measured ACS aperture colors plus the assumed reddening values allow us to predict a reddened $V-I$ color to be compared with ground-based aperture colors. For the 10 of 15 ACS-observed clusters with $(V-I)$ colors (ground-based, mainly from the compilation in Barmby et al. 2000), the agreement between predicted and observed $(V-I)$ colors is very good: the mean offset is $0.02 \pm 0.02$ mag, with a median absolute deviation of 0.06 mag. The cluster B023 has been observed in all three bandpasses discussed here: F606W and F814W with ACS/HRC, and $V$ with STIS. The fits of King (1966) models to these three independent profiles in $\$ 5$ yield extinction-corrected total magnitudes of $F 814 W=12.09, F 606 W=12.88$, and $V=12.99$, with uncertainties of about $\pm 0.02 \mathrm{mag}$ in each. The implied global $(F 606 \mathrm{~W}-F 814 \mathrm{~W})_{0}=0.79$ is essentially identical to the aperture color computed from the measurements in Table 1, and the fitted global $(V-F 606 W)_{0}$ and $(V-F 814 W)_{0}$ are within 0.1 mag of the predicted valuessuggesting a level of agreement similar to that between $(V-I)_{\text {pred }}$ and $(V-I)_{\text {obs }}$ more generally.

The cluster B041 is somewhat unusual. Its integrated colors suggest that it is perfectly average, compared to the rest of the M31 cluster system. However, the ACS image shows that a very bright, red star about 0.5 from the cluster center is responsible for the redness of its $(F 606 W-F 814 W)$ and $(V-I)$ aperture colors. Both the innermost intensities from the ELLIPSE profiles and the total cluster $F 606 W$ and $F 814 W$ magnitudes from model fitting (in which the star is "smoothed over" in some sense), imply a de-reddened $(F 606 W-F 814 W)_{0} \simeq 0.4$ - much bluer than any other cluster in the sample. In fact, the population-synthesis model predictions of C. Maraston suggest that the oldest possible age for such a blue cluster is $\lesssim 1.5$ Gyr. Because of its red integrated color, this cluster is not included in any of the 'massive young M31 cluster' samples of Fusi Pecci et al. (2005) - showing that integrated properties are not always reliable guides to the true nature of extragalactic globular clusters. There are eight clusters in the WFPC2 sample which are members of 'blue luminous compact cluster' samples 'A' or 'B' in Fusi Pecci et al. (2005). These are suspected of being young clusters by virtue of very blue colors $\left[(B-V)_{0}<0.45\right]$ or strong $\mathrm{H} \beta$ absorption. These clusters and $\mathrm{B} 041$ are not included in any of the fundamental plane correlations in $\$ 6.3$, a total of $84 \mathrm{M} 31$ clusters are considered there. 


\section{Models}

Converting luminosity-based parameters from the model fits into mass-based quantities requires knowledge of mass-to-light ratios. Velocity-dispersion measurements do exist for a fraction of the sample (see \$5.3) but the measurements are far from complete or uniform. Instead, as in McLaughlin et al. (2007a), we use population-synthesis models to predict massto-light ratios for the full sample, using the code of Bruzual \& Charlot (2003) and assuming the (disk) stellar IMF of Chabrier (2003). These can then be used to produce (for example) predicted velocity dispersions that can be compared to current and future spectroscopic data. The values of $\Upsilon_{V}^{\text {pop }}$ for an assumed age of 13 Gyr are discussed further and tabulated in $\$ 5.2$ and shown as a histogram in Figure 2. For comparison, also shown is the (re-normalized to the same sample size) distribution of mass-to-light ratios for 85 Galactic globulars with parameters cataloged by McLaughlin \& van der Marel (2005), calculated using the same population-synthesis model code with the same assumed stellar IMF and for the same assumed age of $13 \mathrm{Gyr}$ as in the present analysis. The distribution peaks at a slightly higher value of $\Upsilon_{V}^{\text {pop }}$ in M31 vs. the Milky Way, which simply reflects a slightly higher average GC metallicity in the sample of M31 clusters.

The cluster structural models used in the fits are described in detail by McLaughlin et al. (2007a). The three models considered here are the often-used King (1966) single-mass, isotropic, modified isothermal sphere; an alternate modification of a single-mass, isotropic isothermal sphere, based on a model originally introduced by Wilson (1975) for elliptical galaxies; and the Sérsic (1968) or $R^{1 / n}$ model. Wilson (1975) models are similar to the standard King (1966) models, in that their cores are nearly isothermal, but their envelope structures are relatively more extended - a feature that has been found to better describe the density profiles of old globulars and young massive clusters in the Local Group and in NGC 5128. See McLaughlin \& van der Marel (2005) and McLaughlin et al. (2007a) for more detailed discussion and for general comparisons between the two types of models. Two additional models, 'power-law' profiles and the analytic King (1962) model, are found by McLaughlin et al. (2007a) to provide little or no information beyond that contained in the first three models, and are not discussed here.

Fitting the structural models to the data involves first selecting a value for $r_{0}$, the scale radius, 4 and computing the dimensionless model profile $\widetilde{I}_{\bmod } \equiv I_{\bmod } / I_{0}$. The model is then convolved with the appropriate PSF having parameters taken from Table 3 to yield the product $\widetilde{I}_{\bmod }^{*}\left(R \mid r_{0}\right)$. The fitting procedure allows for a varying scale radius $r_{0}$ and non-zero

\footnotetext{
${ }^{4}$ The definition of $r_{0}$ varies between models: for Sérsic (1968) models, $I\left(r_{0}\right) \equiv 0.5 I_{0}$, while for King (1966) and Wilson (1975) this relation is only approximate. See McLaughlin et al. (2007a) for details.
} 
background $I_{\mathrm{bkg}}$, and so minimizes

$$
\chi^{2}=\sum_{i} \frac{\left[I_{\mathrm{obs}}\left(R_{i}\right)-I_{0} \times \widetilde{I}_{\mathrm{mod}}^{*}\left(R_{i} \mid r_{0}\right)-I_{\mathrm{bkg}}\right]^{2}}{\sigma_{i}^{2}}
$$

for the measured intensity profile $I_{\mathrm{obs}}\left(R_{i}\right)$ and uncertainties $\sigma_{i}$ of each cluster in Table 2 .

Uncertainties on the fitted and derived model parameters are estimated in the usual way, from the range of their values in all models for which $\chi^{2}$ is within some specified distance of the absolute minimum (e.g., $\Delta \chi^{2} \leq 1$ for $68 \%$ confidence intervals). Because the formal error bars estimated by ELLIPSE for the isophotal intensities are artificially small, the best-fit $\chi_{\min }^{2}$ can be exceedingly high ( $\gg N_{\text {pts }}$ ) even when a model fit is actually very good. This would result in unrealistically small estimates of parameter uncertainties. We chose to rescale the $\chi^{2}$ for all fitted models by a common factor chosen to make the global minimum $\chi_{\min }^{2}=\left(N_{\text {pts }}-4\right)$, where $N_{\text {pts }}$ is the number of points used in the model fitting. Under this re-scaling, the global minimum reduced $\chi^{2}$ per degree of freedom is exactly one. This prescription for deciding fit uncertainties does not affect the identification of the best-fitting model itself, since the relative sizes of the uncertainties on individual data points are not changed.

\section{Results}

\subsection{Model-fitting}

Figure 3 shows example surface brightness profiles and model fits for four M31 clusters chosen to span the observed luminosity range. The observed data plotted have had the fitted $I_{\mathrm{bkg}}$ subtracted and then been converted to (extinction-corrected) surface brightnesses. Points with intensities below the subtracted background in the upper panel are represented here as solid points placed on the lower $x$-axis, with error bars extending upwards. The Figure shows that the M31 GCs are well-resolved by the HST imaging, and also demonstrates the core saturation for the brightest clusters exemplified by B151. For these particular clusters there is relatively little difference in the goodness-of-fit of the three different models. As expected, the Wilson (1975) models, with their extended halos, favor somewhat lower background levels, and the 'cuspier' Sérsic (1968) models favor higher central surface brightnesses. The clusters have projected half-light radii $R_{h}=2-4 \mathrm{pc}$ and King-model concentrations in the range 1-1.5, quite typical values for GCs in both M31 (Barmby et al. 2002) and the Milky Way (Harris 1996).

Table 4 summarizes the basic ingredients of all model fits to the full sample. The first 
column in this table gives the cluster name; the second, the detector/filter combination from which the density profile was derived; the third, the extinction in the F606W band; the fourth, the color term to transform photometry from the native bandpass of the data to the standard $V$ scale (see \$3.3), and the fifth column lists the number of points in the intensity profile that are flagged as $\mathrm{OK}$ in Table 2 above, and thus were used to constrain our model fits. Subsequent columns in Table 4 cover three lines for each cluster, one line for each type of model fit. Column (6) identifies the model. Column (7) gives the minimum unreduced $\chi^{2}$ obtained for that class of model (without the re-scaling applied for uncertainty estimation). Column (8) gives the best-fit background intensity. Column (9) gives the dimensionless central potential $W_{0}$ of the best-fitting model (for King 1966 and Wilson models only). Column (10) gives the concentration $c \equiv \log \left(r_{t} / r_{0}\right)$, or the Sérsic index $n$ of the best fit. Column (11) gives the calibrated and extinction-corrected central surface brightness in the native bandpass of the data. Column (12) gives the logarithm of the best-fit scale radius $r_{0}$ in arcsec (see \$4), and Column (13) gives the logarithm of $r_{0}$ in units of pc (obtained from the angular scale assuming $D=780 \mathrm{kpc}$ for M31). Error bars on all these parameters were defined in the same way as McLaughlin et al. (2007a).

\subsection{Derived quantities}

Table 5 contains a number of other structural cluster properties derived from the basic fit parameters. The details of their calculation are given in McLaughlin et al. (2007a); the contents are:

1. $\log r_{t}=c+\log r_{0}$, the model tidal radius in pc (always infinite for Sérsid models)

2. $\log R_{c}$, the projected core radius of the model fitting a cluster, in units of pc

3. $\log R_{h}$, the projected half-light, or effective, radius of a model: that radius containing half the total luminosity in projection, in units of $\mathrm{pd}$

4. $\log \left(R_{h} / R_{c}\right)$, a measure of cluster concentration

5. $\log I_{0}$, the logarithm of the best-fit central $(R=0)$ luminosity surface density in the $V$ band, in units of $L_{\odot, V} \mathrm{pc}^{-2}$

6. $\log j_{0}$, the logarithmic central $(r=0)$ luminosity volume density in the $V$ band (for Sérsid models the density at the 3 -D radius $r_{0}$ ), in units of $L_{\odot} \mathrm{pc}^{-3}$

\footnotetext{
${ }^{5}$ Calculations show that the three-dimensional half-light radius $r_{h}=4 / 3 R_{h}$.
} 
7. $\log L_{V}$, the logarithm of the total integrated model luminosity in the $V$ band

8. $V_{\text {tot }}=4.83-2.5 \log \left(L_{V} / L_{\odot}\right)+5 \log (D / 10 \mathrm{pc})$ is the total, extinction-corrected apparent $V$-band magnitude of a model cluster

9. $\log I_{h} \equiv \log \left(L_{V} / 2 \pi R_{h}^{2}\right)$ is the $V$-band luminosity surface density averaged over the half-light/effective radius, in units of $L_{\odot, V} \mathrm{pc}^{-2}$.

The uncertainties on all of these derived parameters have been estimated (separately for each given model family) following the $\chi^{2}$ re-scaling procedure described above. The recomputed values for the 59 clusters from Barmby et al. (2002), for King (1966) models only, are reported at the end of this Table.

Table [ next lists a number of "dynamical" cluster properties derived from the structural parameters already given plus a mass-to-light ratio. The first two columns of this table contain the name of each cluster and the combination of detector/filter for the observations. Column (3) lists the mass-to-light ratio, in solar units, adopted for each object from the analysis in 84 . The values of $\Upsilon_{V}^{\text {pop }}$ assume an age of 13 Gyr for all clusters. The error bars on $\Upsilon_{V}^{\text {pop }}$ in Table 6 include a \pm 2 -Gyr uncertainty in age, as well as the previously tabulated uncertainties in $[\mathrm{Fe} / \mathrm{H}]$. The remaining entries in Table 6 are, for the best fit of every model type to every cluster:

1. $\log M_{\text {tot }}=\log \Upsilon_{V}^{\text {pop }}+\log L_{V}$, the integrated model mass in solar units, with $\log L_{V}$ taken from Column (10) of Table 5

2. $\log E_{b}$, the integrated binding energy in ergs, defined through $E_{b} \equiv-(1 / 2) \int_{0}^{r_{t}} 4 \pi r^{2} \rho \phi d r$

3. $\log \Sigma_{0}$, the central surface mass density in units of $M_{\odot} \mathrm{pc}^{-2}$

4. $\log \rho_{0}$, the central volume density in units of $M_{\odot} \mathrm{pc}^{-3}$ (for Sérsid models the density at the $3-\mathrm{D}$ radius $r_{0}$ )

5. $\log \Sigma_{h}$, the surface mass density averaged over the effective radius $R_{h}$, in units of $M_{\odot} \mathrm{pc}^{-2}$

6. $\log \sigma_{\mathrm{p}, 0}$, the predicted line-of-sight velocity dispersion at the cluster center (or at $R_{c}$ for Sérsic models), in $\mathrm{km} \mathrm{s}^{-1}$

7. $\log v_{\text {esc }, 0}$, the predicted central "escape" velocity in $\mathrm{km} \mathrm{s}^{-1}$

8. $\log t_{\mathrm{rh}}$, the two-body relaxation time at the model projected half-mass radius, in years 
9. $\log f_{0} \equiv \log \left[\rho_{0} /\left(2 \pi \sigma_{c}^{2}\right)^{3 / 2}\right]$, a measure of the model's central (at $r_{0}$ for Sérsic models) phase-space density or relaxation time, in units of $M_{\odot} \mathrm{pc}^{-3}\left(\mathrm{~km} \mathrm{~s}^{-1}\right)^{-3}$. For $f_{0}$ in these units, and $t_{r c}$ in years, $\log t_{r c} \simeq 8.28-\log f_{0}($ McLaughlin \& van der Marel 2005).

The uncertainties in these derived dynamical quantities are estimated from their variations around the minimum of $\chi^{2}$ on the model grids, as above, combined in quadrature with the uncertainties in the population-synthesis model $\Upsilon_{V}^{\text {pop }}$.

Finally, Table 7 provides the last few parameters required to construct the fundamental plane of globular clusters in M31 under any of the equivalent formulations of it in the literature (see $\$ 6.3$ below). The last three columns of Table 7 are modified versions of the " $\kappa$ " parameters of Bender et al. (1992), who found that early-type galaxies and (separately) globular clusters defined the edges of planes in $\kappa$ space. We define the parameters using mass rather than luminosity surface density, as this is more useful for comparing globulars to younger clusters and galaxies (see McLaughlin et al. 2007b), and emphasize this by using the notation $\kappa_{m, i}$.

$$
\begin{aligned}
\kappa_{m, 1} & \equiv\left(\log \sigma_{\mathrm{p}, 0}^{2}+\log R_{h}\right) / \sqrt{2} \\
\kappa_{m, 2} & \equiv\left(\log \sigma_{\mathrm{p}, 0}^{2}+2 \log \Sigma_{h}-\log R_{h}\right) / \sqrt{6} \\
\kappa_{m, 3} & \equiv\left(\log \sigma_{\mathrm{p}, 0}^{2}-\log \Sigma_{h}-\log R_{h}\right) / \sqrt{3}
\end{aligned}
$$

In calculating $\kappa_{m, 1}, \kappa_{m, 2}$, and $\kappa_{m, 3}$ for Table 7 , the $\sigma_{\mathrm{p}, 0}$ predicted in Table 6 (Column 7) by adoption of population-synthesis mass-to-light ratios has been used, and $\Sigma_{h}$ evaluated by adding $\log \Upsilon_{V}^{\text {pop }}$ to $\log I_{h}$ from Column (12) of Table 5, (This means $\kappa_{m, 3}$ is therefore independent of the assumed mass-to-light ratio.) $R_{h}$ is also taken from Table 5 but put in units of kpc rather than pc, for compatibility with the galaxy-oriented definitions of Bender et al. (1992).

\subsection{Predicted Velocity Dispersions}

One use for the material presented in the previous subsections is to predict observable, line-of-sight velocity dispersions averaged over circular apertures of any radius, which can then be compared directly to extant and forthcoming spectroscopic data on the clusters in the sample. Ultimately such comparisons will be useful not only for independent assessments of the self-consistency of the structural model fits, but also to evaluate how close the dynamical mass-to-light ratios in these old globulars come to the expectations of population-synthesis models with no dark matter.

Given any of the models with a fitted $r_{0}$ and $c$ or $n$ for any cluster, solving Poisson's and Jeans' equations and projecting along the line of sight yields a dimensionless velocity- 
dispersion profile, $\widetilde{\sigma}_{\mathrm{p}}=\sigma_{\mathrm{p}}(R) / \sigma_{\mathrm{p}, 0}$ as a function of projected clustercentric radius $R$. The density-weighted average of $\widetilde{\sigma}_{\mathrm{p}}^{2}$ within a circular region of radius $R_{\text {ap }}$ then gives a dimensionless aperture dispersion for the cluster. Finally, normalizing with the predicted $\sigma_{\mathrm{p}, 0}$ from the fits in Table 6, we can predict an observable $\sigma_{\mathrm{ap}}\left(R_{\mathrm{ap}}\right)$ for every model for every cluster, and for any specified $R_{\text {ap }}$. Table 8 gives the results of these calculations for each of 5 apertures: $R_{\text {ap }}=R_{h} / 8, R_{h} / 4, R_{h}, 4 R_{h}$, and $R_{\text {ap }}=8 R_{h}$. For a distance of $780 \mathrm{kpc}$ to M31, a typical GC $R_{h}=3-4$ pc translates to roughly $0^{\prime \prime} 8-1$.'1, so the range of apertures over which $\sigma_{\text {ap }}$ is calculated should be a good match to reasonable observations. The velocity dispersion inside other apertures can be obtained by interpolation.

These predicted velocity dispersions can already be compared in a limited way to observations. Measured velocity dispersions for 29 M31 GCs, of which 22 are included in the current HST sample, are available from from Cohen (2006), Dubath \& Grillmair (1997), Diorgovski et al. (1997), and Peterson (1989). These are summarized in Table 9. Figure 4 compares observed and model-predicted velocity dispersions, where the model predictions are derived from interpolation on the values in Table 8 to the aperture size reported for

the observations. Aperture sizes were computed as $R_{\mathrm{ap}}=\sqrt{l w / \pi}$ where $l$ and $w$ are the reported spectrograph slit dimensions.

Figure 4 shows that, except for a constant ratio, there is generally good agreement between observed and predicted velocity dispersions (the exception, cluster B037, is discussed further in $\$ 5.5)$. This justifies our use of population synthesis model mass-to-light ratios to derive masses and other properties such as binding energy. This procedure gives us a much larger sample of clusters to work with for a wider variety of analyses than would be possible using only direct velocity dispersion measurements. The median ratio between observed and predicted velocity dispersions is $\sigma_{\text {obs }} / \sigma_{\text {pred }}=0.85$, with an inter-quartile range \pm 0.15 . This corresponds to an ratio between dynamical and population-synthesis-derived mass-to-light ratios of $\Upsilon_{V}^{\text {dyn }} / \Upsilon_{V}^{\text {pop }}=0.73 \pm 0.25$, consistent with the value for this ratio of $0.82 \pm 0.07$ found by McLaughlin \& van der Marel (2005) for Milky Way and old Magellanic Cloud clusters. The lower dynamical mass-to-light ratios compared to those derived from population-synthesis models is consistent with with theoretical expectations that old Milky Way and M31 GCs have lost low-mass stars through evaporation, an effect not included in the models.

\subsection{Comparison of Fits in F606W and F814W}

Comparing model fits to the same cluster observed in different filters allows assessment of the systematic errors and color dependencies in the fits. Figure 5 compares the parameters 
derived from fits to the 15 ACS clusters observed in both F606W and F814W. In general the agreement is quite good, with somewhat higher scatter for the Wilson (1975) and Sérsic (1968) model fits than for the King (1966) fits. There are a few unsuccessful fits with very high concentrations, small sizes and extremely bright central surface brightnesses: the King (1966) model fit to B082 in F606W; Wilson (1975) model fit to B042 in F606W and B063 in F814W; and the Sérsid (1968) model fit to B147 in F814W. These are all clusters in which the central core data are not used due to saturation; fortunately the data in the other filter produce a more reasonable-looking fit. For all three models, the central $V$-band surface brightnesses as fit to the $\mathrm{F} 814 \mathrm{~W}$ data are slightly fainter than those from the F606W data, while the total model luminosities are essentially the same. The latter implies that our estimated $(V-F 606 W)$ and $(V-F 814 W)$ colors in $\$ 3.3$ are correct, while the former implies that the cluster centers are slightly bluer than the average cluster color. This could indicate the presence of blue stragglers or blue horizontal branch stars in these massive clusters, but it could also be due to the differing levels of saturation. A broader range of data will be needed to investigate the issue of color gradients in M31 GCs more thoroughly (for a discussion of color gradients in Milky Way clusters, see Djorgovski \& Piotto 1993).

Because the model fit results in the two ACS bands are quite similar, and also because for the STIS and WFPC2 data we have only $V$-band data, we do not consider the F814W model fits in the following analyses. Parameters derived from the model fits given in Tables 5$]$ refer to the F606W band observations only. The exception is B082, where we substitute the F814W fit results for the King (1966) fit.

\subsection{Comparison to previous results for individual clusters}

The famous M31 cluster G001 has discrepant published values of its half-light radius (see Barmby et al. 2002; Ma et al. 2006b), which was a major motivation for re-analyzing it in this paper. Meylan et al. (2001) reported a three-dimensional $r_{h}=3$ ".7, which converts to a projected $R_{h}=2 \prime \prime 8$, while Barmby et al. (2002) measured $R_{h}=0^{\prime \prime} 82$ and Rich et al. (1996) $R_{h}=0.706$ Our King (1966) model fits to the ACS/HRC F555W data resulted in a halflight radius $R_{h}=0^{\prime \prime} 85$, and our Wilson (1975) fit to the same data yielded $R_{h}=1^{\prime \prime} \cdot 2$. The Meylan et al. (2001) value is clearly the outlier, likely because it is for the half-mass, rather than the half-light radius, and because the Mevlan et al. (2001) analysis used multi-mass King (1966) models. The surface brightness profile from the HRC observations is almost

\footnotetext{
6 Larsen (2001) pointed out that the integrated photometry given by Mevlan et al. (2001) implies a half-light radius of about 1 "'18, much closer to other values.
} 
identical to that presented in Mevlan et al. (2001), which combined data from WFPC2 observations including those used by Rich et al. (1996), so we conclude that the physical model assumptions used are responsible for the differences between authors. Recently, Ma et al. (2007) also analyzed the ACS/HRC data used here: these authors derived a higher concentration $c=2.01 \pm 0.02$ compared to our King (1966) model value of 1.77 , and a value of $R_{h}=1^{\prime \prime} .73 \pm 0$ '.07 about twice as large as ours. This appears to be the result of weighting the outermost points in the surface brightness profile more heavily, yielding a larger tidal radius and higher concentration. The dashed line in Ma et al.'s Figure 3, which represents their model fit with the outermost surface brightness points excluded, is quite similar to our model fit, and we believe this to be more robust particularly in the better-defined inner part of the cluster.

The heavily-reddened M31 cluster B037 has also been the subject of intensive study. Ma et al. (2006b) analyzed the same F606W image of B037 discussed here, fitting a King (1962) model to a surface brightness profile made from a PSF-deconvolved image. They derived a core radius $r_{0}=0^{\prime \prime} .72$, half-light radius $r_{h}=1^{\prime \prime}$. 11 , concentration $c=0.91$, and central surface brightness $\mu(0)=17.21 \mathrm{mag} \operatorname{arcsec}^{-2}$ in F606W [using our values for extinction and $V-F 606 W$ color, this becomes $\mu_{V, 0}=13.49$.] Our model fits generally result in a somewhat higher concentration and smaller core radius $\left[r_{0}=0^{\prime \prime} .56, r_{h}=1\right.$.'09, $c=1.23$ for the King (1966) model]. This relatively small change would not likely have affected Ma et al.'s conclusions about the nature of this cluster.

Ma et al. (2006a) predict a velocity dispersion of $72 \mathrm{~km} \mathrm{~s}^{-1}$ for B037, on the basis of a mass estimate of $\approx 3 \times 10^{7} M_{\odot}$. However, these $M$ and $\sigma$ values are based on a populationsynthesis $M / L_{V}$ ratio applied to an intrinsic cluster luminosity obtained by assuming a distance of $\sim 950 \mathrm{kpc}$ to M31, instead of our adopted $780 \mathrm{kpc}$ (and a large $E(B-V)$ close to the one we have used). Thus, comparison of their numbers with ours requires first multiplying their derived mass by $(780 / 950)^{2}=2 / 3$. In addition, the Ma et al. mass and velocity dispersion are actually calculated for what the cluster would have been at an age of 10 Myr. However, the same population-synthesis models that they (like us) usedi.e., Bruzual \& Charlot (2003) - show that the cumulative effects of supernova explosions, massive-star winds, and AGB mass loss over 12-13 Gyr lead to a reduction of the 10Myr total cluster mass by another factor of $\simeq 2$ at its current, old age (see also, e.g., Fioc \& Rocca-Volmerange 1997). Therefore, the present-day mass of B037 implied by the work of $\mathrm{Ma}$ et al. is in fact $\approx 1 \times 10^{7} M_{\odot}$, in good agreement with our results here (Table [6).

The high velocity dispersion predicted for B037 by Ma et al. is a 'global' (not central) value following from the virial theorem assuming both their high mass and a rough estimate of the cluster half-light radius ( $\simeq 2.6 \mathrm{pc}$ in their analysis), which is significantly smaller than 
that in our more accurate profile fitting $\left(R_{h}=3.9\right.$ pc; e.g., Table 8 , column 5$)$. Scaling to a 3 times smaller present-day mass, as above, and to a $\sim 1.5$-times larger $R_{h}$, the expected global velocity dispersion following self-consistently from Ma et al. is $72 \mathrm{~km} \mathrm{~s}^{-1} \times \sqrt{(1 / 3) / 1.5}=$ $34 \mathrm{~km} \mathrm{~s}^{-1}$, which is then entirely consistent with our predicted $\sigma_{\text {ap }}$ inside the largest apertures in Table 8 .

Cohen (2006) has measured a velocity dispersion of $19.6 \pm 3.5 \mathrm{~km} \mathrm{~s}^{-1}$ for B037 within an aperture of $R_{\text {ap }} \simeq 1.6$ arcsec. Our predicted dispersion within this aperture follows from interpolation on Table 8: $38.3 \pm 2.7 \mathrm{~km} \mathrm{~s}^{-1}$. This factor-of-two discrepancy (which implies a factor-of-four discrepancy between the true cluster mass and that estimated from our de-reddened total luminosity and 13-Gyr old population-synthesis $M / L_{V}$ ratio) could be resolved by lowering the mass-to-light ratio, but reducing $\Upsilon_{V}^{\text {pop }}$ by a factor of 4 would require imply an age for B037 of only a few Gyr, in conflict with the SED-fitting results. Reducing the reddening of B037 to $E(B-V) \sim 0.85$ would yield a total luminosity in better agreement with the measured $\sigma_{v}$, but this would also conflict with the Ma et al. (2006a) SED-fitting. The only viable resolution appears to be the suggestion by Cohen (2006) that the dust lane projected across the face of the cluster (reported by Ma et al. 2006b) distorts measurements of extinction from integrated photometry. This should be testable with color profiles and star-count analysis from the ACS images. Such analysis is beyond the scope of the present paper, and the properties of B037 computed using $E(B-V)=1.36$ are used in our subsequent analysis.

\subsection{Quality of Fit for Different Models}

McLaughlin \& van der Marel (2005) and McLaughlin et al. (2007a) give detailed discussions of the differences between various cluster models and their applicability to GCs in galaxies other than M31. Which structural model best represents the M31 clusters? Figure6 compares the $\chi^{2}$ values for the model fits as a function of cluster size $R_{h}$ and total (model) luminosity $L_{V}$. The difference between models is parameterized as

$$
\Delta \equiv\left(\chi_{\text {alternate }}^{2}-\chi_{\text {King }}^{2}\right) /\left(\chi_{\text {alternate }}^{2}+\chi_{\text {King }}^{2}\right)
$$

which is independent of the $\chi^{2}$ re-scaling; significantly better fits of one model over another are signaled by $|\Delta| \gtrsim 0.2$. In general, King (1966) models fit the M31 cluster data better than Wilson (1975) or Sérsid (1968) models. Cluster size does not strongly affect $\Delta$, but luminosity does: King (1966) models are more strongly preferred for more luminous M31 clusters. The preference for King (1966) models for M31 clusters differs from results using the same profile-fitting software for for clusters in the Milky Way, Magellanic Clouds, and 
Fornax dSph (McLaughlin \& van der Marel 2005) and NGC 5128 clusters (McLaughlin et al. 2007a), where Wilson (1975) models fit as well as or better than than King (1966) models. The results for M31 clusters do not appear to depend on the image saturation for some clusters - the clusters observed with ACS/HRC, where saturation was not a problem, were also better-fit with King (1966) models. The fitting procedure returned higher background levels for M31 clusters than those in NGC 5128, but the background value does not correlate with $\Delta$.

Figure 7 compares the values of cluster parameters derived from King (1966) and Wilson (1975) model fits. The parameters do not clearly vary with goodness-of-fit, and even the major outliers are not always much better fit with one model or the other. The mean offsets between parameters derived for the same cluster from King (1966) and Wilson (1975) models are comparable to the rms scatter, and only slightly larger than the typical parameter uncertainties: $\delta \mu_{V, 0}=0.04 \pm 0.18, \delta\left(\log R_{h}\right)=0.14 \pm 0.13, \delta\left(\log L_{V}\right)=0.08 \pm 0.06$, and $\delta\left(\log R_{c}\right)=0.03 \pm 0.02$. Small-scale systematic differences between the models (for example, Wilson (1975) models, which have more extended halos, always return fainter central surface brightnesses $\mu_{V, 0}$ than King (1966) models) do not affect the global cluster parameters, such as the binding energy $E_{b}$ and total luminosity $L_{V}$. These are very similar in all three of King (1966), Wilson (1975) and Sérsid (1968) models. We suspect that the preference for King (1966) over Wilson (1975) models is due to some more subtle feature of the observational data that we have not yet been able to isolate, rather than an intrinsic difference between M31 clusters and those in other galaxies. However, since a majority of the M31 clusters have parameters derived only with King (1966) model fits, we use these models as the basis for the fundamental plane analysis presented in $\$ 6.3$. We do not expect the following discussion to depend on the model choice.

\section{Discussion}

We now combine the M31 GC parameters newly derived in the previous section with those for clusters in the Milky Way and Magellanic Clouds (McLaughlin \& van der Marel 2005) and NGC 5128 (McLaughlin et al. 2007a) to form a large sample of GCs that have been analyzed in a nearly homogeneous way. The sample comprises 291 clusters in six galaxies, an unprecedentedly large sample for defining the GC fundamental plane. In the following analysis, cluster luminosities are derived from the model fits to the surface photometry rather than integrated or aperture measurements. For the M31 and NGC 5128 clusters, model and measured luminosities are found to have excellent correspondence, while for Milky Way and Magellanic Cloud clusters, discrepancies between model and measured luminosities are 
often attributable to the measurement aperture being smaller than the clusters' full size (McLaughlin \& van der Marel 2005). Where M31 clusters are compared with NGC 5128 clusters from McLaughlin et al. (2007a), the 27 clusters omitted from that analysis are also omitted here. As discussed above, King (1966) model parameters are adopted for clusters in all galaxies.

\subsection{Ellipticity distribution}

There are several possible explanations for the elongation of globular clusters: Larsen (2001) lists elongation as possibly resulting from galaxy tides, internal rotation, cluster mergers (crossing times in star cluster complexes are short; Fellhauer \& Kroupa 2005) and 'remnant elongation' (due to accretion?) from some clusters' former lives as dwarf galaxy nuclei. The latter two mechanisms would seem to be applicable to only a small fraction of clusters, probably the more massive one. They could be related to the observations that the most luminous young and old LMC clusters tend to be more flattened, and that the brightest globular clusters in both the Milky Way and M31 are also the most flattened (van den Bergh \& Morbey 1984; van den Bergh 1996).7 Measurement of GC ellipticities is an uncertain business particularly for faint clusters: the shape of outer isophotes is affected by the galaxy background light and low signal-to-noise. For the extragalactic clusters measured with ELLIPSE, very low values of ellipticity are disfavored because the ELLIPSE algorithm diverges at $\epsilon=0$. With these caveats in mind, it is still worthwhile to explore ellipticity distributions and correlations with other parameters for clues to the origin of GC flattening.

Testing the idea that GC elongation is due to galaxy tides can be done to some extent by comparing the ellipticities of clusters in different galaxies, and thus different dynamical environments. Figure 8 shows the distribution of ellipticities for clusters in the Milky Way (Harris 1996), M31 (this work), and NGC 5128 (Harris et al. 2006). Compared to Figure 7 of Harris et al. (2002), the number of M31 clusters is about 10\% larger (although the sample is somewhat different and includes more very luminous clusters); the number of NGC 5128 clusters is about 3 times larger and the distribution of their ellipticities somewhat more skewed to lower values. The distributions of ellipticities for M31 and NGC 5128 are not statistically different; both differ from the Milky Way distribution in having few very round clusters (likely an artifact of the measurement technique). The bottom panel of the Figure shows the distribution of ellipticity with galactocentric position: no correlation is evident.

\footnotetext{
${ }^{7}$ As discussed in 95.5 , there is some controversy about whether G001 or B037 is the most luminous M31 GC, but both are quite elliptical.
} 
From this comparison there is no clear evidence for major differences in ellipticity distribution between the three different galaxies' GCs, and thus no evidence that the overall galaxy environment is a major factor.

If cluster ellipticities are caused by internal processes such as rotation or velocity anisotropy, then relaxation through dynamical evolution should act to reduce any initial flattening (Fiestas et al. 2006). More dynamically-evolved clusters might be expected to be rounder (although the relaxation time is long in the outer regions of clusters which heavily influence measured ellipticity). Figure 9] shows ellipticity as a function of luminosity and half-mass relaxation time for clusters in the three galaxies. A mild systematic decrease in $\epsilon$ with increased $L$ is visible, though with considerable scatter; measurements of very large ellipticities for clusters with low luminosities are more likely to be spurious. While the observation of van den Bergh (1996) that the most luminous clusters in M31 and the Milky Way are highly elliptical remains true, it is clear that all three galaxies also have very luminous clusters which are quite round: did these bright, round clusters simply have less angular momentum at formation? Or are they the 'true' globular clusters while the more flattened luminous clusters are remnant dwarf galaxy nuclei somehow flattened while being removed from their original galaxies? Further modeling of the dwarf galaxy stripping process might help to answer this question. No correlation of $\epsilon$ with the more important dynamical quantity $t_{r h}$ is evident: the data do not appear to favor the contention that more evolved clusters are rounder. While primordial angular momentum still seems to be the most straightforward explanation for GC ellipticity, the observed distribution appears to be due to a number of factors.

\subsection{Correlations with position and metallicity}

Structures of GCs in the Milky Way have been shown to be largely independent of galactocentric distance and metallicity (Djorgovski \& Mevlan 1994; Diorgovski 1995; McLaughlin 2000), except for the correlation of half-light radius with $R_{\mathrm{gc}}$ first noted by van den Bergh et al. (1991). Studies of cluster structures in nearby galaxies (Barmby et al. 2002; Harris et al. 2002) have found similar results, with the exception of a mild correlation between cluster half-light radius and metallicity: more metal-rich clusters tend to be smaller. The same effect is seen in more distant galaxies where the clusters' structures are barely resolved. Brodie \& Strader (2006) discuss several possible explanations and conclude that the $R_{h}-[\mathrm{Fe} / \mathrm{H}]$ correlation is most likely a consequence of the correlation between $R_{h}$ and $R_{\mathrm{gc}}$ and the tendency of metal-rich GCs to be located at smaller $R_{\mathrm{gc}}$.

Figure 10 plots structural parameters and $[\mathrm{Fe} / \mathrm{H}]$ for our sample as a function of galac- 
tocentric distance $R_{\mathrm{gc}}$. For the Milky Way clusters the true three-dimensional distance is used, while for external galaxies only projected distances are available. For the M31 clusters, no correlation of concentration $c$ with $R_{\mathrm{gc}}$ is seen. Central surface brightness $\mu_{V, 0}$ becomes fainter with increasing $R_{\mathrm{gc}}$ but this is likely to be a selection effect: fainter clusters are difficult to see against the bright background of the M31 bulge (McLaughlin et al. 2007b discuss the same effect in detail for NGC 5128). As expected, $R_{h}$ increases with $R_{\mathrm{gc}}$, although the trend is largely driven by the innermost clusters in M31. In the Milky Way the $R_{h}-R_{\mathrm{gc}}$ trend is driven both by small inner clusters and the large clusters at very large distances from the Galactic center. Such distant, low-surface brightness clusters will be difficult to detect in external galaxies, and their absence from the present sample may well be due to selection effects. For the clusters in NGC 5128, which cover a more restricted range in $R_{\mathrm{gc}}$, McLaughlin et al. (2007b) conclude that the $R_{h}-R_{\mathrm{gc}}$ trend is weak and only marginally significant. For the M31 and Milky Way clusters, a weak correlation of $[\mathrm{Fe} / \mathrm{H}]$ with $R_{\mathrm{gc}}$ is present, but again is driven mainly by clusters at the extremes of $R_{\mathrm{gc}}$. The Magellanic Cloud and Fornax dSph clusters are offset from the larger galaxies in all of these plots, as might be expected from the parent galaxies' smaller sizes. Scaling cluster properties by galactocentric distance as in McLaughlin (2000) is therefore expected to be useful in computing the 'fundamental plane' for these clusters.

There are half a dozen very small $\left(R_{h} \lesssim 1 \mathrm{pc}\right)$ M31 clusters visible in the upper right panel of Figure 10, Such clusters are not found in the other galaxies studied, which makes their presence in M31 slightly suspicious. Of the six clusters, only the largest, B155, has a confirmatory radial velocity (Galleti et al. 2006) while the remainder are classified by those authors as "cluster candidates". The compactness of these objects makes assessing their nature problematic, even with the use of HST imaging. They are a reminder that the structural analysis of star clusters in distant galaxies will always be problematic: existing catalogs are neither fully complete nor entirely reliable.

Figure 11 plots cluster structural parameters as a function of $[\mathrm{Fe} / \mathrm{H}]$; only clusters with measured values of $[\mathrm{Fe} / \mathrm{H}]$ (not assumed average values) are shown. No correlation of concentration $c$ with $[\mathrm{Fe} / \mathrm{H}]$ is seen. A weak correlation of $R_{h}$ with $[\mathrm{Fe} / \mathrm{H}]$ is present in the expected sense: $R_{h}$ decreases with increased metallicity. The exception is NGC 5128, for which the best-fit slope of $\log R_{h}$ against $[\mathrm{Fe} / \mathrm{H}]$ is zero; the present sample of clusters in this galaxy also has a lower metallicity gradient $\left([\mathrm{Fe} / \mathrm{H}]\right.$ vs. $\left.R_{\mathrm{gc}}\right)$ than in other galaxies. The $R_{h}-[\mathrm{Fe} / \mathrm{H}]$ corrlation weakens if only clusters with $2<R_{\mathrm{gc}}<20 \mathrm{kpc}$ are considered, suggesting that a different mix of bulge and halo clusters in M31 and the Milky Way compared to NGC 5128 may be responsible. For central surface brightness $\mu_{V, 0}$ there is a slight systematic increase with $[\mathrm{Fe} / \mathrm{H}]$, likely because of the weak correlation of $[\mathrm{Fe} / \mathrm{H}]$ and $R_{\mathrm{gc}}$. Finally, the lower-right panel shows that there is a weak mass-metallicity correlation: this is due entirely to our use 
of population synthesis model mass-to-light ratios, which increase rapidly above $[\mathrm{Fe} / \mathrm{H}]=-1$ (see Figure 1 of McLaughlin et al. 2007b). All of these trends, however, are mild compared with the correlations with cluster luminosity to be presented below.

Figure 12 shows some of the correlations between cluster parameters and luminosity, for GCs in M31, the Milky Way, NGC 5128, the Magellanic Clouds (MCs), and the Fornax dSph. The properties of clusters in all six galaxies fall in the same regions of parameter space. The selection effects discussed above are apparent in that there are fewer faint and low-surfacebrightness clusters in the extragalactic samples. The new M31 ACS/STIS observations in particular do not contain any faint, low-concentration clusters, partly because the ACS observations targeted luminous M31 clusters and also because very low-concentration M31 clusters are too well-resolved to be analyzed with the ellipse-fitting method used (see $\S$ 3.1). There are few very large $\left(R_{h}>10 \mathrm{pc}\right)$ extragalactic clusters, probably because these are difficult to recognize and more easily confused with background galaxies. The right-hand panels of the Figure show the lack of correlation between $R_{h}$ and $L$ and the strong correlation of $E_{b}$ with $L$ which are both hallmarks of the globular cluster fundamental plane. The fundamental plane correlations evidently hold over nearly 4 decades in luminosity and in a variety of environments.

\subsection{The Fundamental Plane}

Correlations between globular cluster structural parameters are often summarized as describing a "fundamental plane" analogous to (but different from) that for elliptical galaxies. There are several equivalent formulations of the globular cluster fundamental plane in

the literature (Burstein et al. 1997; Djorgovski 1995; McLaughlin 2000; McLaughlin et al. $2007 \mathrm{~b})$. Here we concentrate on the relationships between half-light radius, mass, and binding energy to search for differences between the fundamental planes of clusters in different galaxies. An important caveat to this analysis is that the GC masses used are derived from population synthesis model-predicted mass-to-light ratios, not from directly observed velocity dispersions (see $\S 5.3$ ).

One of the many ways of looking at the globular cluster fundamental plane is a plot of projected cluster half-light radii as a function of mass. Modulo some stretching, this plot is equivalent to one of projected half-light surface density $\Sigma_{h}$ versus $M$, which in turn is the largest part of the $\kappa_{m, 1}$ and $\kappa_{m, 2}$ parameter space. A different reformulation makes $R_{h}$ vs. $M$ equivalent to binding energy $E_{b}$ vs. $M: E_{b}=f(c) M^{2} / R_{h}$ where $f(c)$ is a weak function of the cluster concentration (McLaughlin 2000, and see below). Figure 13 shows $R_{h}$ versus $M$ for GCs in all of our galaxies. As is well known from previous investigations of the Galactic GC 
system (van den Bergh et al. 1991; Diorgovski \& Mevlan 1994), there is no clear, monotonic mass-radius relation for globular clusters. However, at masses $M \gtrsim 1.5 \times 10^{6} M_{\odot}$ there is an apparent upturn in the lower envelope of $R_{h}$ versus $M$. McLaughlin et al. (2007b) interpret this increasing lower bound on $R_{h}$ for clusters as an extension of a very similar relation for early-type galaxies. The latter relation follows from the existence of a "zone of exclusion" in the (luminosity-based) $\kappa$ space of Burstein et al. (1997), which can be interpreted as a mass-dependent upper limit on the average surface density $\Sigma_{h}$. The bound $R_{h}^{\min }(M)=$ $\left[M / 2 \pi \sum_{h}^{\max }\right]^{1 / 2}$ for galaxies, simply extrapolated down into the GC mass range, is drawn as the dash-dot line in Figure 13, see (McLaughlin et al. 2007b) for further details.

The two dashed lines in the lower-left corner of the Figure represent a different bound on GC size: evaporation by two-body relaxation, which is expected to occur within 20-40 initial half-mass relaxation times (e.g. Gnedin \& Ostriker 1997). The two lines correspond to $t_{\mathrm{rh}}=(13 / 20)$ Gyr (upper) and $t_{\mathrm{rh}}=(13 / 40)$ Gyr (lower); clusters which formed with masses and sizes to the lower-left of these lines are expected to have evaporated by the present day. Several of the M31 clusters are below the 'evaporation' lines, indicating that they must have evolved into this region of the diagram and may in fact be dissolving. The most extreme of these are the very compact M31 clusters discussed in $\S$ 6.2. The identfication of these objects as M31 GCs is somewhat suspect: high-resolution spectroscopic observations would be of interest to confirm their status and measure velocity dispersions.

None of the galaxies' clusters show evidence for correlation of half-light radius with mass, but there are differences between galaxies in both position and scatter of their clusters in Figure 13. The larger scatter for Milky Way clusters compared to those in M31 and NGC 5128 can be attributed to the presence of more very diffuse clusters in the MW sample (such clusters are difficult to detect in the extragalactic systems; McLaughlin et al. 2007b) and greater distance uncertainties for individual Galactic GCs. The M31 sample has very few diffuse clusters due to the selection effects described above, and so is offset to smaller average $R_{h}$ than the Milky Way or NGC 5128 samples The Magellanic Cloud and Fornax cluster samples are complete, however, and have larger average $R_{h}$ than the large-galaxy GCs.

Is the difference between cluster sizes in different galaxies due to local environment? The structure of tidally-limited clusters should depend on the local galaxy density, and cluster sizes are known to be correlated with galactocentric position (see the upper right panel of Figure 10). A better indicator of galactic potential than galactocentric distance alone is:

$$
R_{\mathrm{gc}}^{*} \equiv \frac{R_{g c} /(8 \mathrm{kpc})}{V_{c} /\left(220 \mathrm{~km} \mathrm{~s}^{-1}\right)}
$$


where $V_{c}$ is the galaxy circular velocity 8 We use values $V_{c}$ in $\mathrm{km} \mathrm{s}^{-1}$ of: Milky Way, 220; M31, 230 (Carignan et al. 2006); NGC 5128, 190 (Woodley 2006; Peng et al. 2004a.b; Mathieu et al. 1996); LMC, 65 (van der Marel et al. 2002); SMC, 60 (Stanimirović et al. 2004), and Fornax dSph, 18 (Walker et al. 2006). For a galaxy whose total mass distribution is an isothermal sphere, mass density $\rho \propto\left(V_{c} / R_{\mathrm{gc}}\right)^{2}$, so $R_{\mathrm{gc}}^{*} \propto \rho^{-1 / 2}$ is a guide to the local galaxy density. Figure 14 shows $R_{h}$ as a function of re-normalized galactocentric distance $R_{\mathrm{gc}}^{*}$, with the four galaxies plotted separately. The normalization has removed the $R_{h} / R_{\mathrm{gc}}$ offset between the Magellanic Cloud/Fornax clusters and those in the large galaxies, indicating that the larger cluster sizes in the small galaxies can be understood as due to the lower galactic densities. The least-squares fit of $\log R_{h}=C+\gamma \log \left(R_{\mathrm{gc}}^{*}\right)$ is shown for each galaxy, and given in Table 10. As might be expected, there is less dependence of $R_{h}$ on $R_{\mathrm{gc}}^{*}$ for the extragalactic clusters compared to the Milky Way GCs, due to the use of projected galactocentric distances in computing $R_{\mathrm{gc}}^{*}$.

The normalization by galactic potential should allow us to fairly compare the fundamental planes of clusters in the different galaxies. In analogy with McLaughlin (2000) and Harris et al. (2002) we define the re-normalized quantities

$$
R_{h}^{*} \equiv R_{h} \times\left(R_{g c}^{*}\right)^{-\gamma}
$$

and

$$
E_{b}^{*} \equiv E_{b} \times\left(R_{g c}^{*}\right)^{\gamma}
$$

where $\gamma$ is derived from the fits given above. Figure 15 shows that there is no correlation of $R_{h}^{*}$ with $[\mathrm{Fe} / \mathrm{H}]$, so any differences between galaxies are not due to residual effects of metallicity on the fundamental plane.

The top panel of Figure 16 shows $E_{b}^{*}$ versus $M$, with the the corresponding least-squares fits for each galaxy given in Table 10. The basic proportionality $E_{b} \propto M^{2}$ is built into the definition of $E_{b}$, and clusters in all galaxies follow this relation very tightly. In particular, the fits for NGC 5128 and Milky Way clusters are identical within their errors, confirming the Harris et al. (2002) result with a sample three times larger and extending to much lower luminosities. At the high end of the mass range, the clusters in both M31 and NGC 5128 fall below the best-fit lines, indicative of the 'break' in cluster properties at $\sim 1.5 \times 10^{6} \mathrm{M}_{\odot}$ noted by McLaughlin et al. (2007b). Below this break, the fits for all galaxies are similar, with the largest differences at the low-mass end where the number of clusters is small and

\footnotetext{
${ }^{8}$ The normalization of $R_{\mathrm{oc}}^{*}$ ensures that quantities derived from it are equivalent to those plotted for Milky Way clusters in McLaughlin (2000). It should properly be computed with the three-dimensional galactocentric distance, but only the projected distance is available for the extragalactic clusters.
} 
measurement errors are large. The bottom panel of Figure 16 shows the difference between $E_{b}^{*}$ predicted from the Milky Way fit and the measured values. Table 10 gives the mean offsets for each galaxy. The NGC 5128 and Magellanic Cloud clusters (and the Milky Way clusters, by construction) have offsets consistent with zero, while the M31 clusters have a slight offset which can be attributed to sampling issues: the lack of diffuse clusters and the presence of the low- $R_{h}$ objects at $M \sim 5 \times 10^{4} \mathrm{M}_{\odot}$

Globular clusters have a strikingly similar nature in the widely different environments we have sampled, including a dwarf spheroidal, irregulars, large disk galaxies, and a giant elliptical. The homogeneous and internally precise data we can now work with, in addition to the much larger sample sizes of clusters, have established the trend of the fundamental plane securely, showing that globular clusters in all these environments follow the first-order trend $E_{b} \sim M^{2}$ quite accurately. The scatter around this basic relation is surprisingly small, showing that the structural properties of these star clusters are far simpler than we could have expected from theoretical arguments alone. Even the most massive GCs seem to show a fairly smooth transition between the properties of clusters and those of dwarf galaxies.

\section{Summary}

New Hubble Space Telescope observations are used to construct surface brightness profiles for 34 M31 globular clusters, including the most massive clusters in the galaxy. Structural models are fit to the surface brightness profiles: for reasons not yet understood, M31 clusters are unlike those in other nearby galaxies in slightly preferring King (1966) models over the more extended Wilson (1975) models. When combined with previously-published data, we now have a comprehensive and publicly available compilation of a complete suite of structural and dynamical parameters for as many GCs in M31 as in the Milky Way. The structural parameters of clusters in M31 and other local galaxies define a very tight fundamental plane with similar slopes $E_{b} \sim M^{2}$, showing the essential similarity of clusters in different environments over a range of almost 4 decades in luminosity or mass. M31 clusters are slightly offset from the $E_{b}-M$ relation defined by the Milky Way and NGC 5128, likely because of sampling effects. In both M31 and NGC 5128 clusters, the most massive GCs trend towards dwarf galaxies in the binding-energy/mass relation at about $10^{6} \mathrm{M}_{\odot}$. The detailed modeling made possible by high-resolution imaging shows that the overall properties of globular clusters in different galaxies are remarkably similar; the subtle differences noted here may point to differences in the histories or environments of their parent galaxies. The present dataset pushes our assessment of the fundamental plane beyond the previous state of the field (McLaughlin 2000; Harris et al. 2002; Barmby et al. 2002; McLaughlin \& van der Marel 
2005), making it possible to search for second-order trends in the FP line and probe the transition between the structures of globular clusters and galaxies.

We thank the referee for a rapid and thoughtful report. Support to P.B. and D.E.M. for program GO-10260 was provided by NASA through a grant from the Space Telescope Science Institute, which is operated by the Association of Universities for Research in Astronomy, Inc., under NASA contract NAS 5-26555. W.E.H. and G.L.H.H. thank the Natural Sciences and Engineering Research Council of Canada for financial support. D.A.F. thanks the Australian Research Council for financial support.

\section{REFERENCES}

Anderson, J. \& King, I. 2005, HST Instrument Science Report ACS 2004-15, Tech. rep., Space Telescope Science Institute

Barmby, P., Holland, S., \& Huchra, J. P. 2002, AJ, 123, 1937

Barmby, P. \& Huchra, J. P. 2001, AJ, 121, 2458

Barmby, P., Huchra, J. P., Brodie, J. P., Forbes, D. A., Schroder, L. L., \& Grillmair, C. J. 2000, AJ, 119, 727

Barmby, P., Perrett, K. M., \& Bridges, T. J. 2002, MNRAS, 329, 461

Baumgardt, H., Makino, J., Hut, P., McMillan, S., \& Portegies Zwart, S. 2003, ApJ, 589, L25

Bender, R., Burstein, D., \& Faber, S. M. 1992, ApJ, 399, 462

Brodie, J. P. \& Strader, J. 2006, ARA\&A, 44, 193

Bruzual, G. \& Charlot, S. 2003, MNRAS, 344, 1000

Burstein, D. \& Heiles, C. 1984, ApJS, 54, 33

Burstein, D., Bender, R., Faber, S., \& Nolthenius, R. 1997, AJ, 114, 1365

Cardelli, J. A., Clayton, G. C., \& Mathis, J. S. 1989, ApJ, 345, 245

Carignan, C., Chemin, L., Huchtmeier, W. K., \& Lockman, F. J. 2006, ApJ, 641, L109

Chabrier, G. 2003, PASP, 115, 763 
Cohen, J. 2006, ApJ, 653, L21

Djorgovski, S. \& Piotto, G. 1993, in "Structure and Dynamics of Globular Clusters", ASP. Conf. Ser. 50, 203

Djorgovski, S. \& Meylan, G. 1994, AJ, 108, 1292

Djorgovski, S. 1995, ApJ, 438, L29

Djorgovski, S. G., Gal, R. R., McCarthy, J. K., Cohen, J. G., de Carvalho, R. R., Meylan, G., Bendinelli, O., \& Parmeggiani, G. 1997, ApJ, 474, L19

Dubath, P. \& Grillmair, C. J. 1997, A\&A, 321, 379

Fellhauer, M. \& Kroupa, P. 2005, MNRAS, 359, 223

Fioc, M. \& Rocca-Volmerange, B. 1997, A\&A, 326, 950

Fiestas, J., Spurzem, R. \& Kim, E. 2006, MNRAS, 373, 677

Ford, H. et al. 2002, SPIE, 4854, 81

Fusi Pecci, F., Bellazzini, M., Buzzoni, A., De Simone, E., Federici, L., \& Galleti, S. 2005, AJ, 130, 554

Galleti, S., Federici, L., Bellazini, M., Buzzoni, A., \& Fusi Pecci, F. 2006, A\&A, 456, 985

Gebhardt, K., Rich, R. M., \& Ho, L. C. 2002, ApJ, 578, L41

—. 2005, ApJ, 634, 1093

Gnedin, O.Y. \& Ostriker, J. E. 1997, ApJ, 474, 223

Haşegan, M., et al. 2005, ApJ, 627, 203

Harris, W. E. 1996, AJ, 112, 1487

Harris, W. E. 2003, in A Decade of Hubble Space Telescope Science, 78-100

Harris, W. E., Harris, G. L. H., Barmby, P., McLaughlin, D. E., \& Forbes, D. A. 2006, AJ, 132,2187

Harris, W. E., Harris, G. L. H., Holland, S. T., \& McLaughlin, D. E. 2002, AJ, 124, 1435

Hilker, M. \& Richtler, T. 2000, A\&A, 362, 895 
Huchra, J. P., Brodie, J. P., \& Kent, S. M. 1991, ApJ, 370, 495

Huxor, A. P., Tanvir, N. R., Irwin, M. J., Ibata, R., Collett, J. L., Ferguson, A. M. N., Bridges, T., \& Lewis, G. F. 2005, MNRAS, 360, 1007

King, I. R. 1962, AJ, 67, 471

-. 1966, AJ, 71, 64

Larsen, S. S. 2001, AJ, 122, 1782

Larsen, S. S., Brodie, J. P., Sarajedini, A., \& Huchra, J. P. 2002, AJ, 124, 2615

Ma, J., de Grijs, R., Yang, Y., Zhou, X., Chen, J., Jiang, Z., Wu, Z., \& Wu, J. 2006a, MNRAS, 368, 1443

Ma, J., van den Bergh, S., Wu, H., Yang, Y., Zhou, X., Chen, J., Wu, Z., Jiang, Z., \& Wu, J. 2006b, ApJ, 636, L93

Ma, J., de Grijs, R., Chen, D., van den Bergh, S., Fan, Z., Wu, Z., Wu, H., Zhou, X., Wu, J., Jiang, Z., \& Chen, J. 2007, MNRAS, in press (astro-ph/0702012)

Maraston, C. 1998, MNRAS, 300, 872

-. 2005, MNRAS, 362, 799

Mathieu, A., Dejonghe, H., \& Hui, X. 1996, A\&A, 309, 30

McLaughlin, D. E. 2000, ApJ, 539, 618

McLaughlin, D. E., Barmby, P., Harris, W. E., Forbes, D. A. \& Harris, G. L. H. 2007a, MNRAS, submitted

McLaughlin, D. E., Harris, W. E., Barmby, P., Forbes, D. A., \& Harris, G. L. H. 2007b, MNRAS, submitted

McLaughlin, D. E. \& van der Marel, R. P. 2005, ApJS, 161, 304

Meylan, G., Sarajedini, A., Jablonka, P., Djorgovski, S. G., Bridges, T. J., \& Rich, R. M. 2001, AJ, 112, 830

Peng, E. W., Ford, H. C. \& Freeman, K. C. 2004, ApJ, 602, 685

Peng, E. W., Ford, H. C. \& Freeman, K. C. 2004, ApJ, 602, 705 
Perrett, K. M., Bridges, T. J., Hanes, D. A., Irwin, M. J., Brodie, J. P., Carter, D., Huchra, J. P., \& Watson, F. G. 2002, AJ, 123, 2490

Peterson, R. 1989, in Dynamics of Dense Stellar Systems, ed. D. Merritt (Cambridge: Cambridge University Press), 161

Rich, R. M., Mighell, K. J., Freedman, W. L., \& Neill, J. D. 1996, AJ, 111, 768

Sérsic, J.-L. 1968, Atlas de Galaxias Australes (Cordoba: Observatoria Astronomico)

Sirianni, M. et al. 2005, PASP, 117, 1049

Stanimirović, S., Staveley-Smith, L. \& Jones, P. A. 2004, ApJ, 604, 176

Trudolyubov, S., \& Priedhorsky, W. 2004, ApJ, 616, 821

Tsuchiya, T., Dinescu, D. I., \& Korchagin, V. I. 2003, ApJ, 589, L29

van den Bergh, S. 1996, The Observatory, 116, 103

van den Bergh, S., Morbey, C., \& Pazder, J. 1991, ApJ, 375, 594

van den Bergh, S. \& Morbey, C. L. 1984, ApJ, 283, 598

van der Marel, R. P., Alves, D. R., Hardy, E., \& Suntzeff, N. B. 2002, AJ, 124, 2639

Walker, M. G., Mateo, M., Olszewski, E. W., Bernstein, R., Wang, X., \& Woodroofe, M. 2006, AJ, 131, 2114

Wilson, C. P. 1975, AJ, 80, 175

Woodley, K. A. 2006, AJ, 132, 2424

This preprint was prepared with the AAS IATEX macros v5.2. 

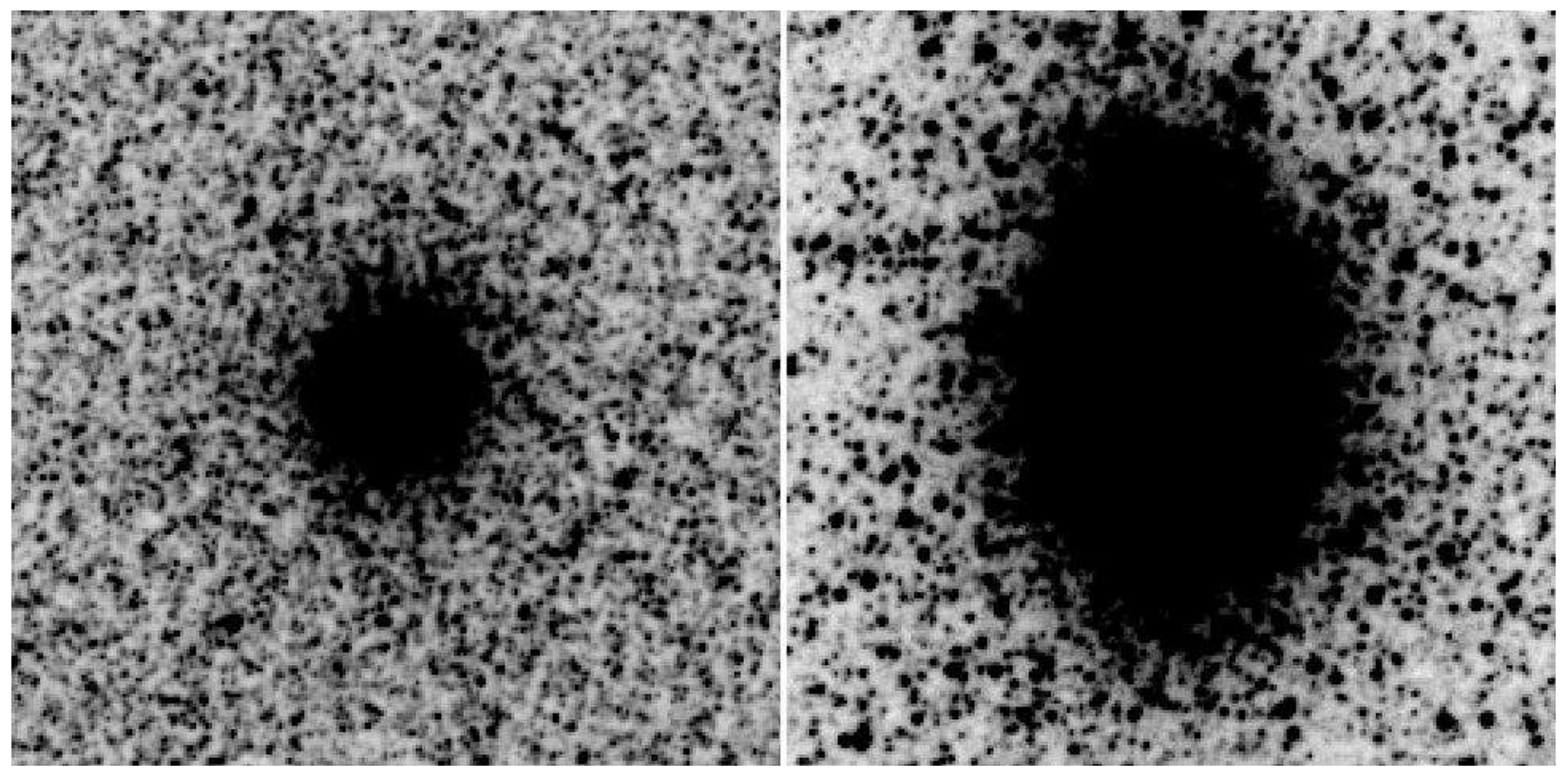

Fig. 1. - ACS/WFC F606W images of two M31 clusters. Left: B147, (classified as a star by Dubath \& Grillmair 1997, based on a velocity dispersion measurement). Right: the very elliptical $(\epsilon=0.28)$ cluster B088. Both images have north up and east to the left and are $12^{\prime \prime}$ square. 


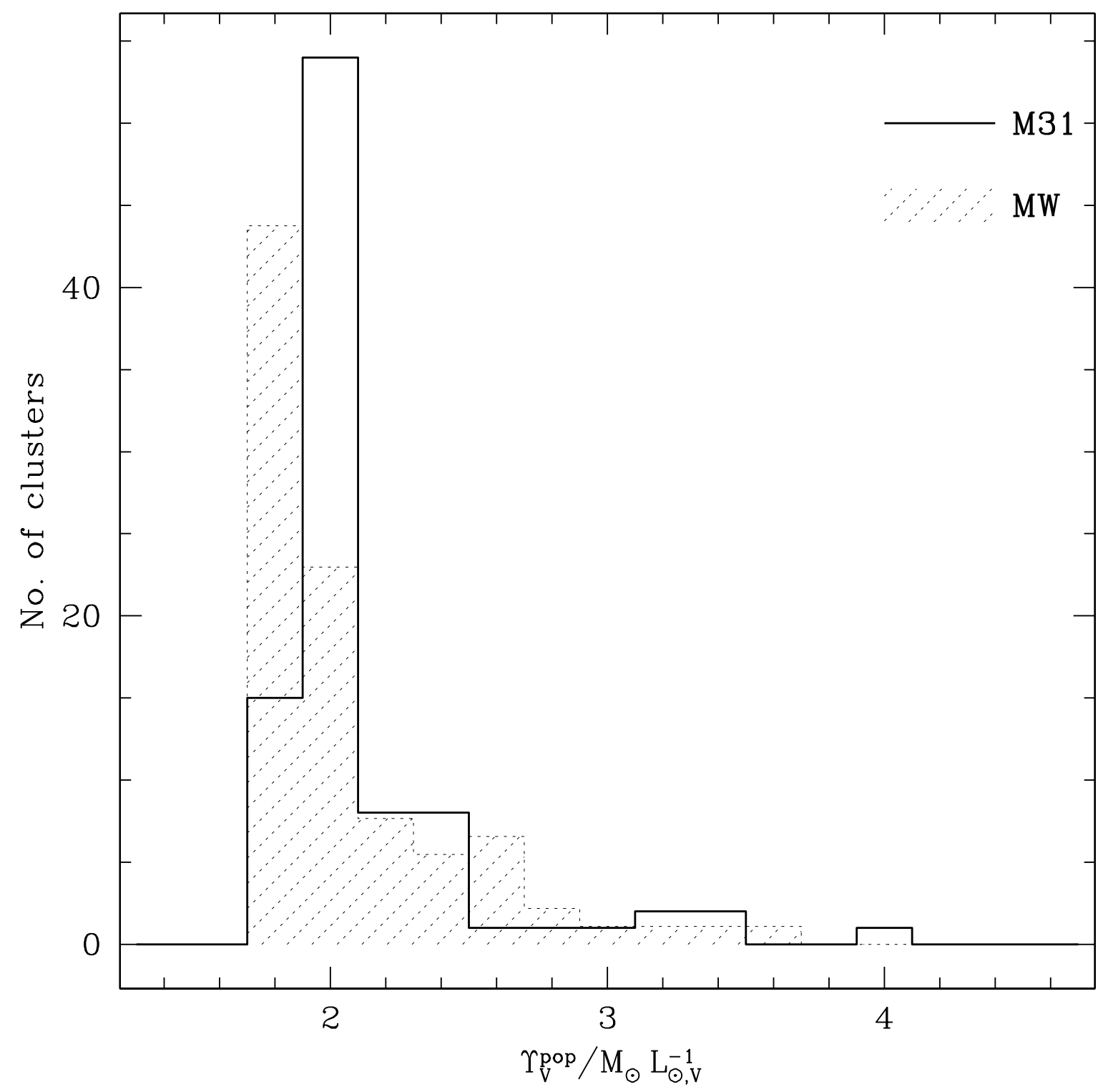

Fig. 2.- Histogram of population-synthesis $V$-band mass-to-light ratios for M31 GCs assuming an age of $13 \mathrm{Gyr}$; and the same for Galactic globulars, from McLaughlin \& van der Marel (2005). 


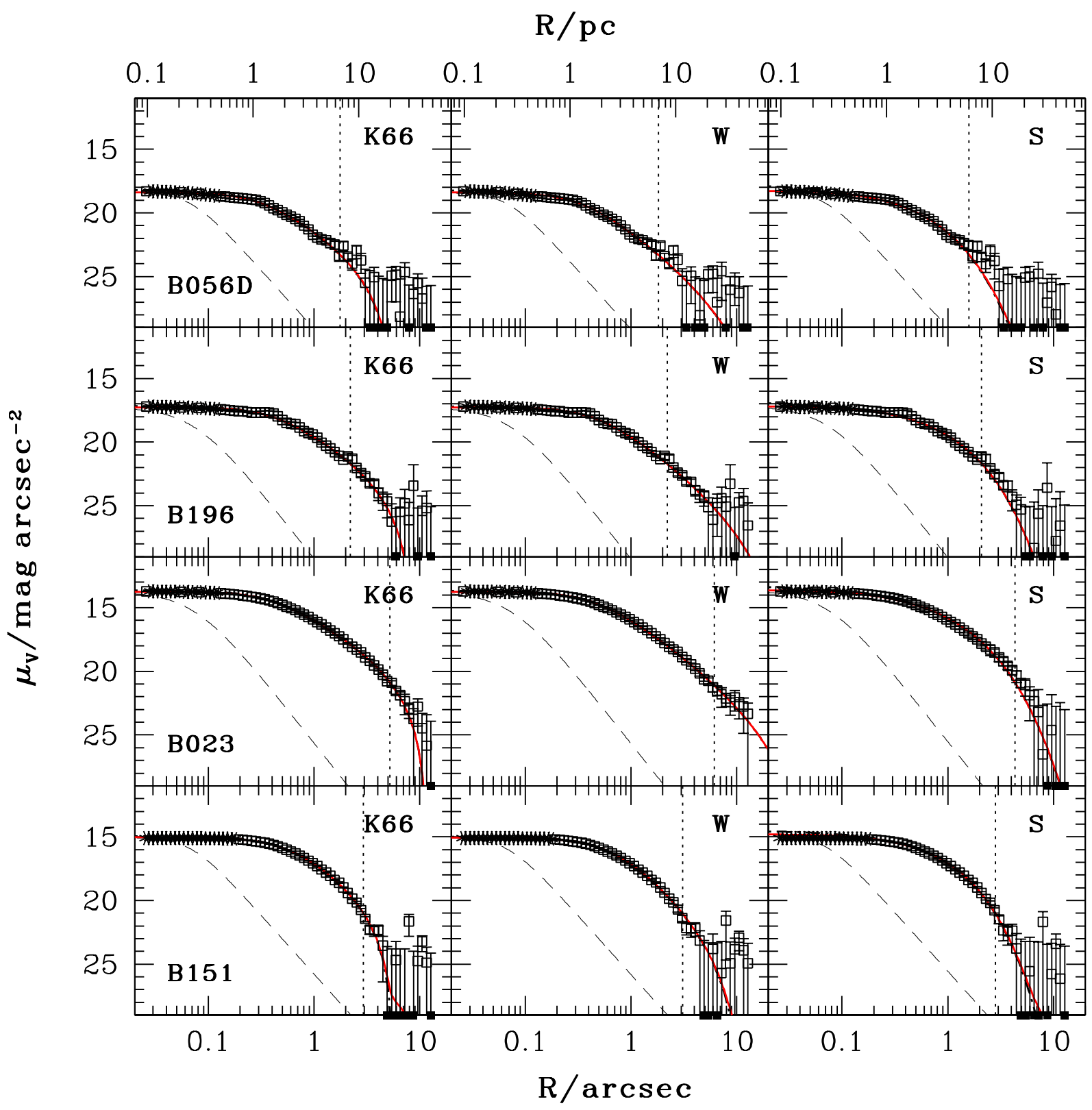

Fig. 3.- Surface brightness profiles and model fits to four M31 GCs. Clusters are in order of increasing luminosity, from top to bottom: B056D, B196, B023, and B151. The three panels for each cluster are, from left to right: King (1966), Wilson (1975) and Sérsic (1968) models. Dashed curves trace the PSF intensity profiles and solid (red) curves the PSF-convolved best-fit models with added background. Vertical dotted lines mark the radius where the best-fit cluster intensity is equal to the background. Open squares are ELLIPSE data points included in the least-squares model fitting and the asterisks are points (flags of BAD, SAT, or DEP in Table 21) not used to constrain the fits. 

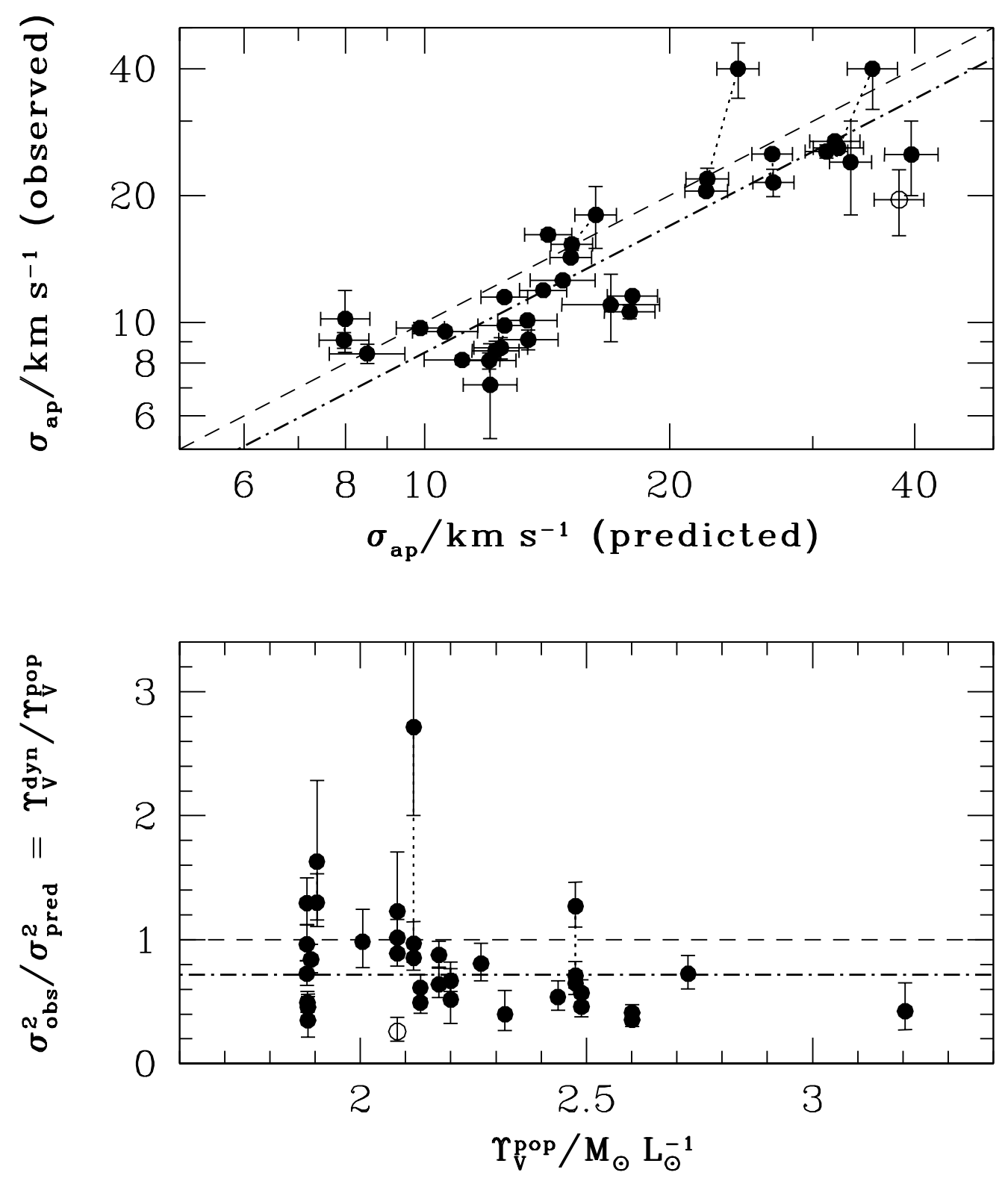

Fig. 4.- Top: comparison of structural-model predictions for central velocity dispersion with observations from Djorgovski et al. (1997), Dubath \& Grillmair (1997), and Peterson (1989). Open symbol is cluster B037, discussed in \$5.5, Points connected by short-dashed lines are duplicate observations of the same cluster. Long-dashed line is line of equality; dot-dashed line is median ratio $\sigma_{\mathrm{obs}} / \sigma_{\text {pred }}=0.85$. Bottom: comparison of dynamical and population-synthesis derived mass-to-light ratios. Long-dashed line is line of equality; dotdashed line is median ratio $\Upsilon_{V}^{\text {dyn }} / \Upsilon_{V}^{\text {pop }}=0.725$. 

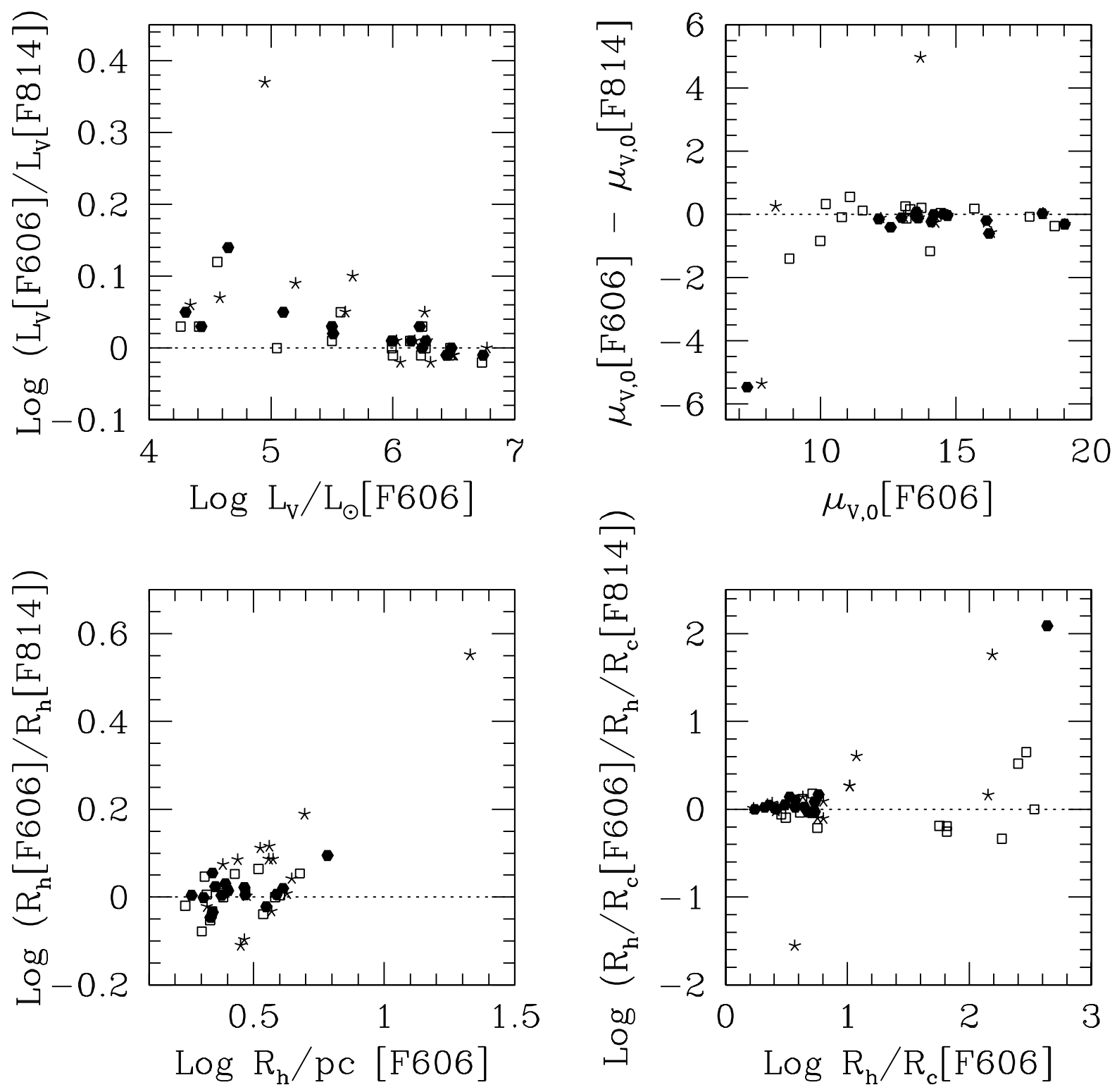

Fig. 5.- Comparison of parameters for model fits to 15 M31 clusters observed in both F606W and F814W filters. Top left: model total magnitude, top right: central surface brightness, bottom left: projected half-light radius, and bottom right: ratio of half-light to core radii (a measure of concentration). Filled circles: King (1966) models, squares: Sérsic (1968) models, stars: Wilson (1975) models. 


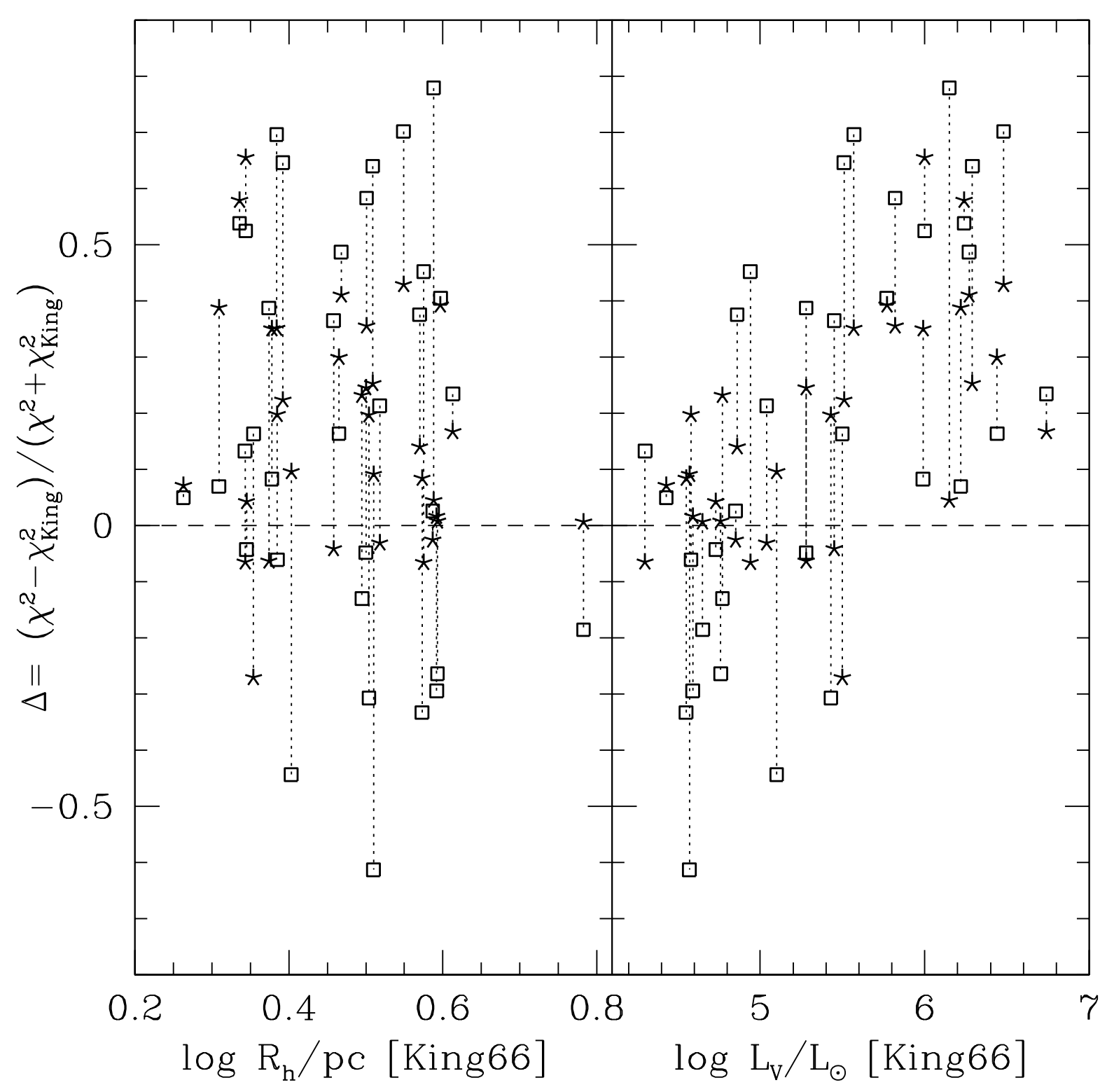

Fig. 6. - Comparison of goodness of M31 cluster model fits as a function of King (1966) model size $R_{h}$ and luminosity $L_{V}$. Models are compared with $\Delta \equiv\left(\chi_{\text {alternate }}^{2}-\right.$ $\left.\chi_{\text {King }}^{2}\right) /\left(\chi_{\text {altennate }}^{2}+\chi_{\text {King }}^{2}\right)$ which is positive if the King (1966) model is a better fit. Asterisks are Wilson (1975) models and open squares Sérsic (1968) models. 

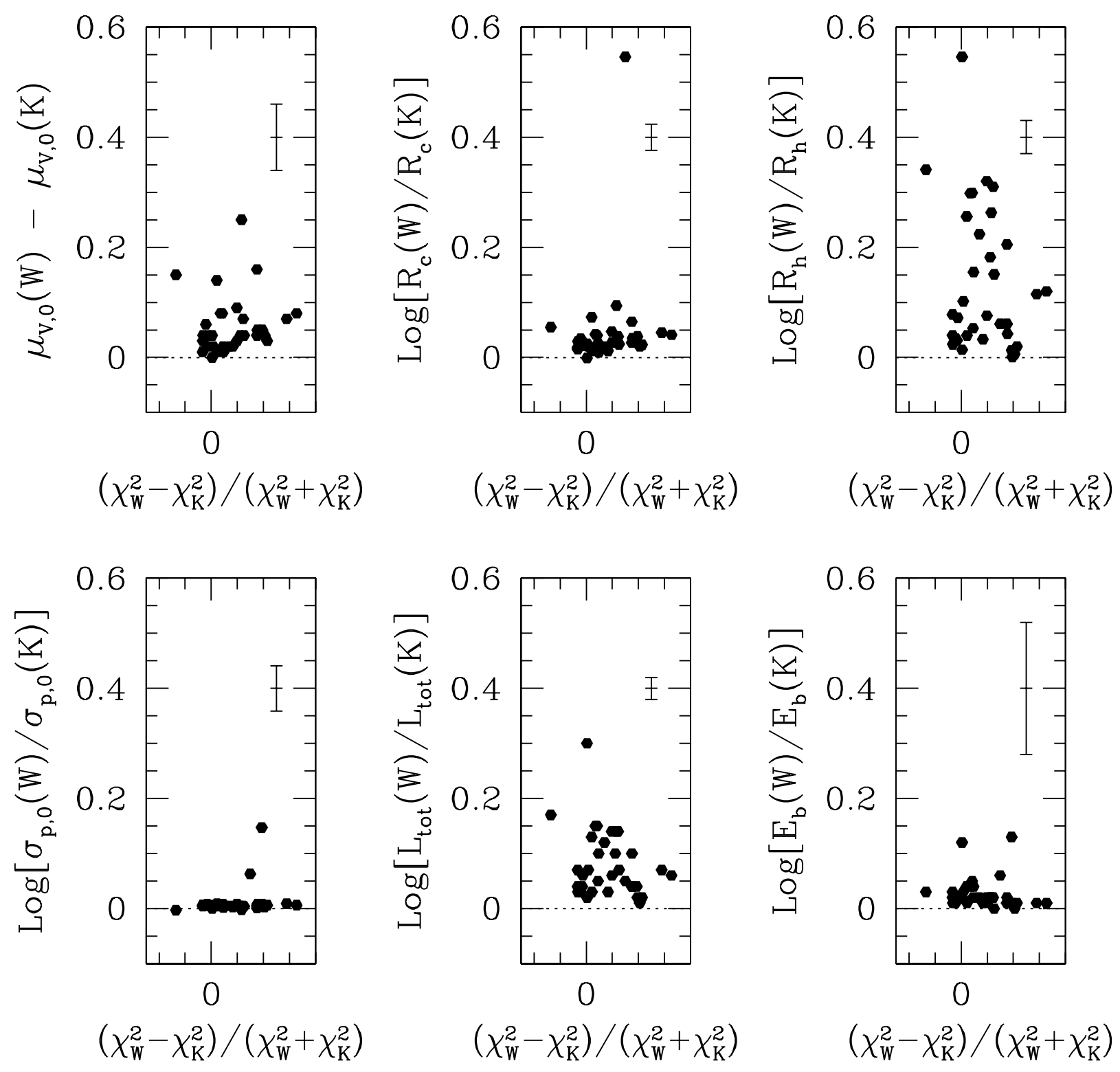

Fig. 7.- Comparison of Wilson (1975) and King (1966) model-fit parameters for M31 clusters as a function of goodness-of-fit difference $\Delta=\left(\chi_{\text {Wilson }}^{2}-\chi_{\text {King }}^{2}\right) /\left(\chi_{\text {Wilson }}^{2}+\chi_{\text {King }}^{2}\right)$. Errorbars show typical parameter uncertainties in a single model fit. 


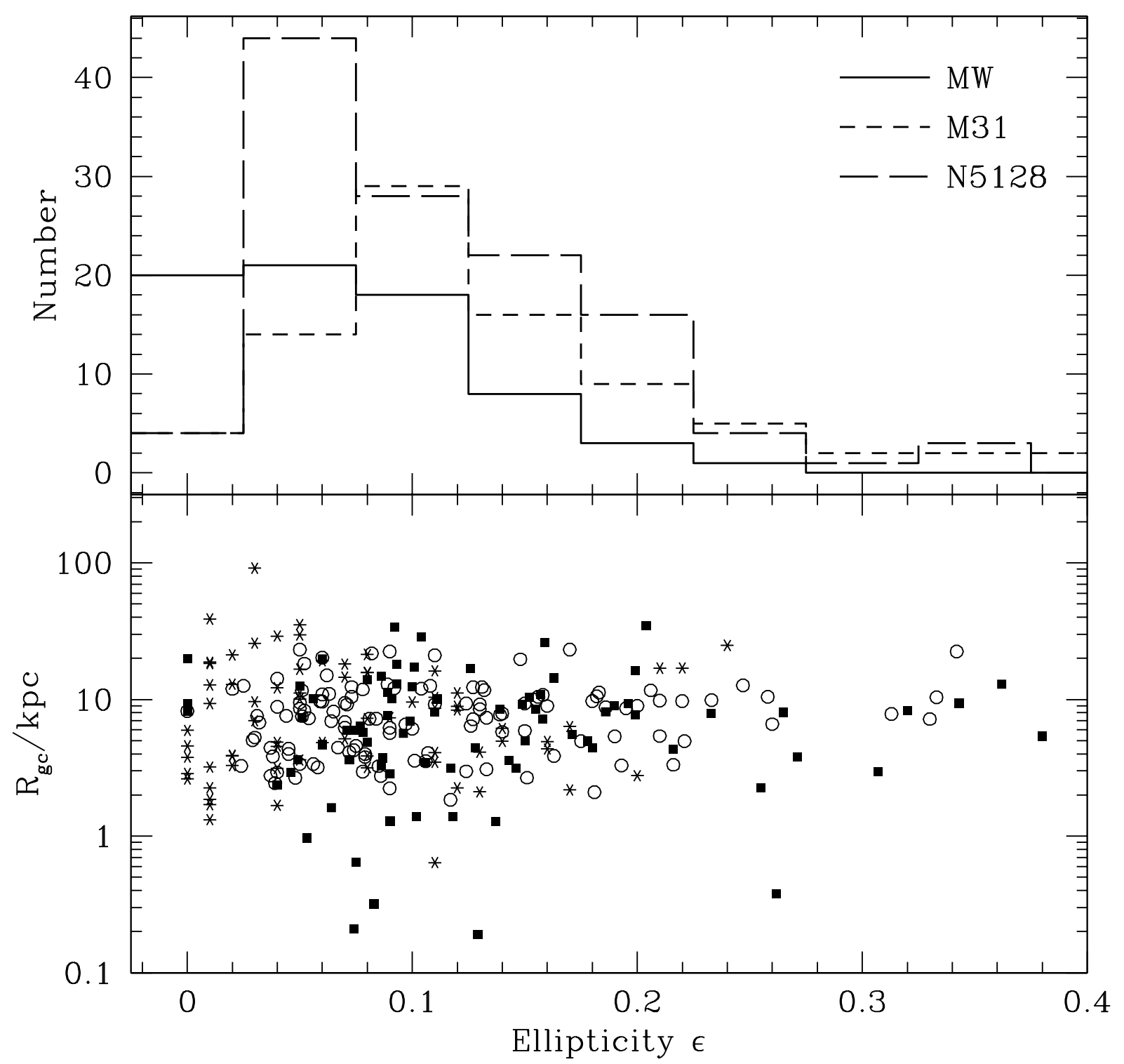

Fig. 8.- Top: distribution of ellipticity for globular clusters in the Milky Way (solid line), M31 (short-dashed lines), and NGC 5128 (long-dashed lines). Bottom: ellipticity versus projected galactocentric radius for globular clusters in M31 (filled squares), NGC 5128 (open circles), and the Milky Way (stars; three-dimensional $R_{\mathrm{gc}}$ used). 


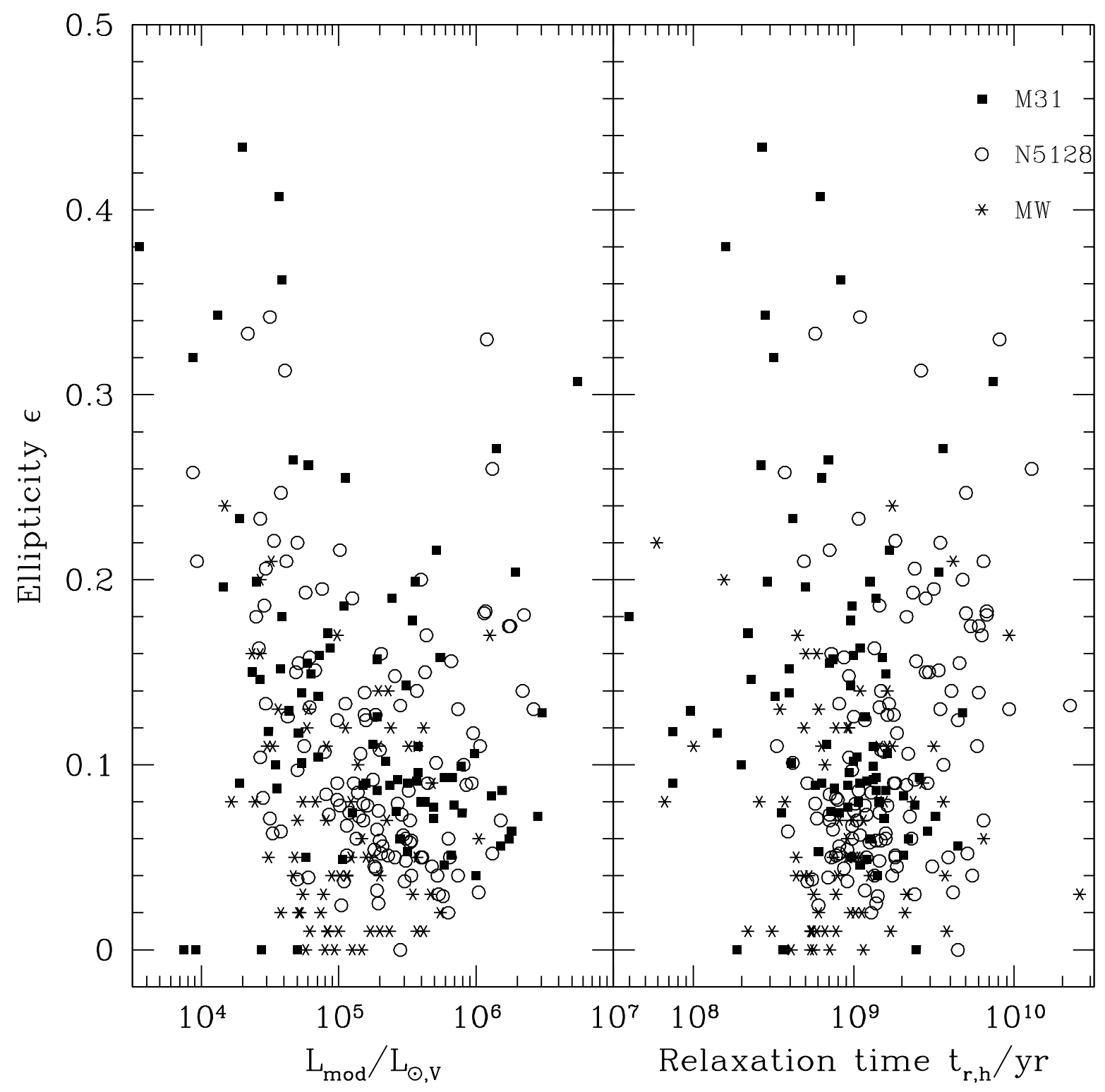

Fig. 9.- Ellipticity versus luminosity and half-mass relaxation time for globular clusters in M31 (filled squares), NGC 5128 (open circles), and the Milky Way (stars). 

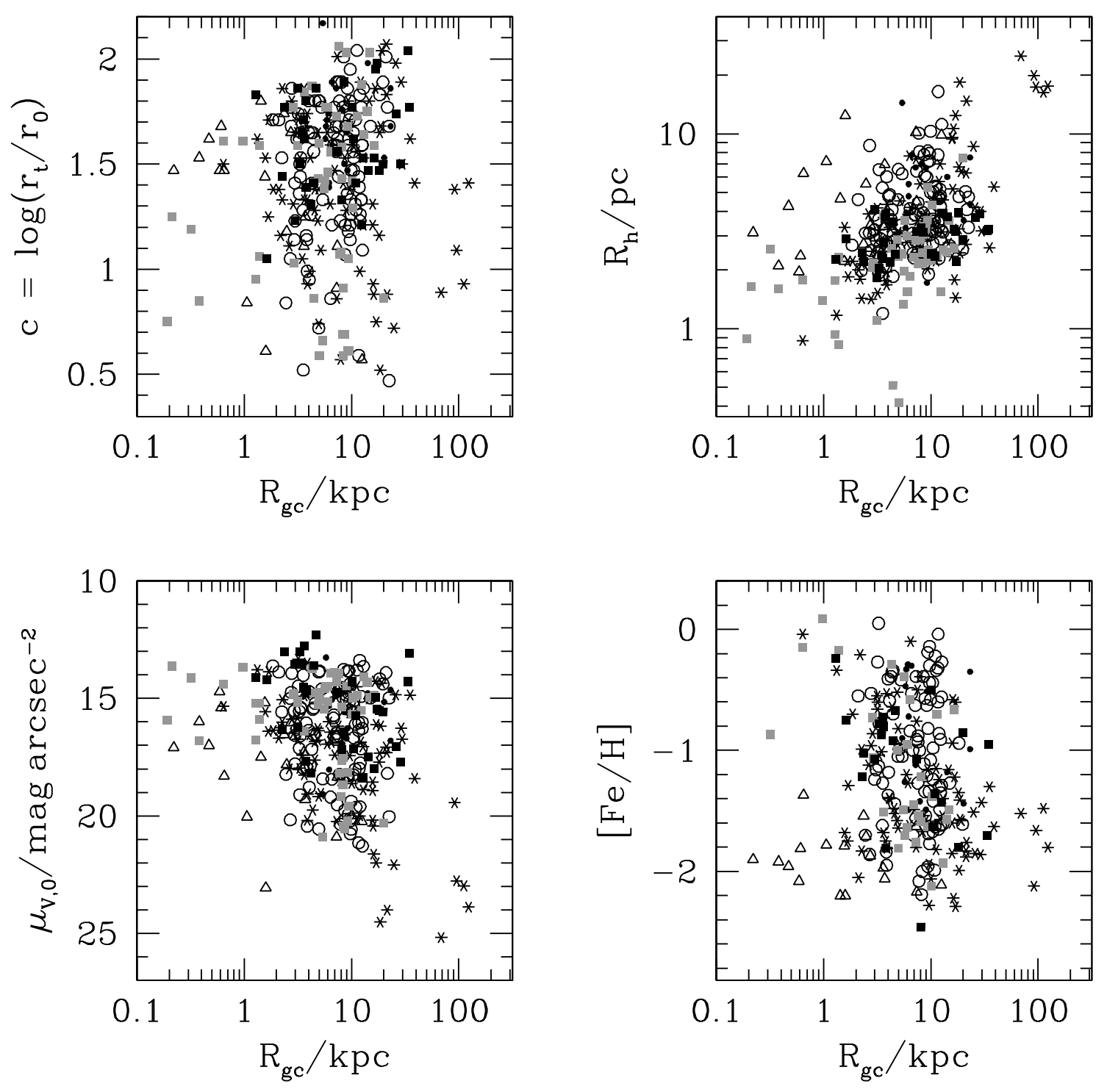

Fig. 10. - Structural parameters as a function of galactocentric distance $R_{\mathrm{gc}}$ for globular clusters in the Milky Way (stars), the Magellanic Clouds and Fornax dwarf spheroidal (open triangles), NGC 5128 [open circles are clusters from McLaughlin et al. 2007a; small filled circles are from Harris et al. 2002], and M31 (filled squares). The darker filled squares are the M31 clusters observed with ACS or STIS; lighter squares are WFPC2 observations. $R_{\mathrm{gc}}$ is three-dimensional for Milky Way clusters but projected two-dimensional for other galaxies. 

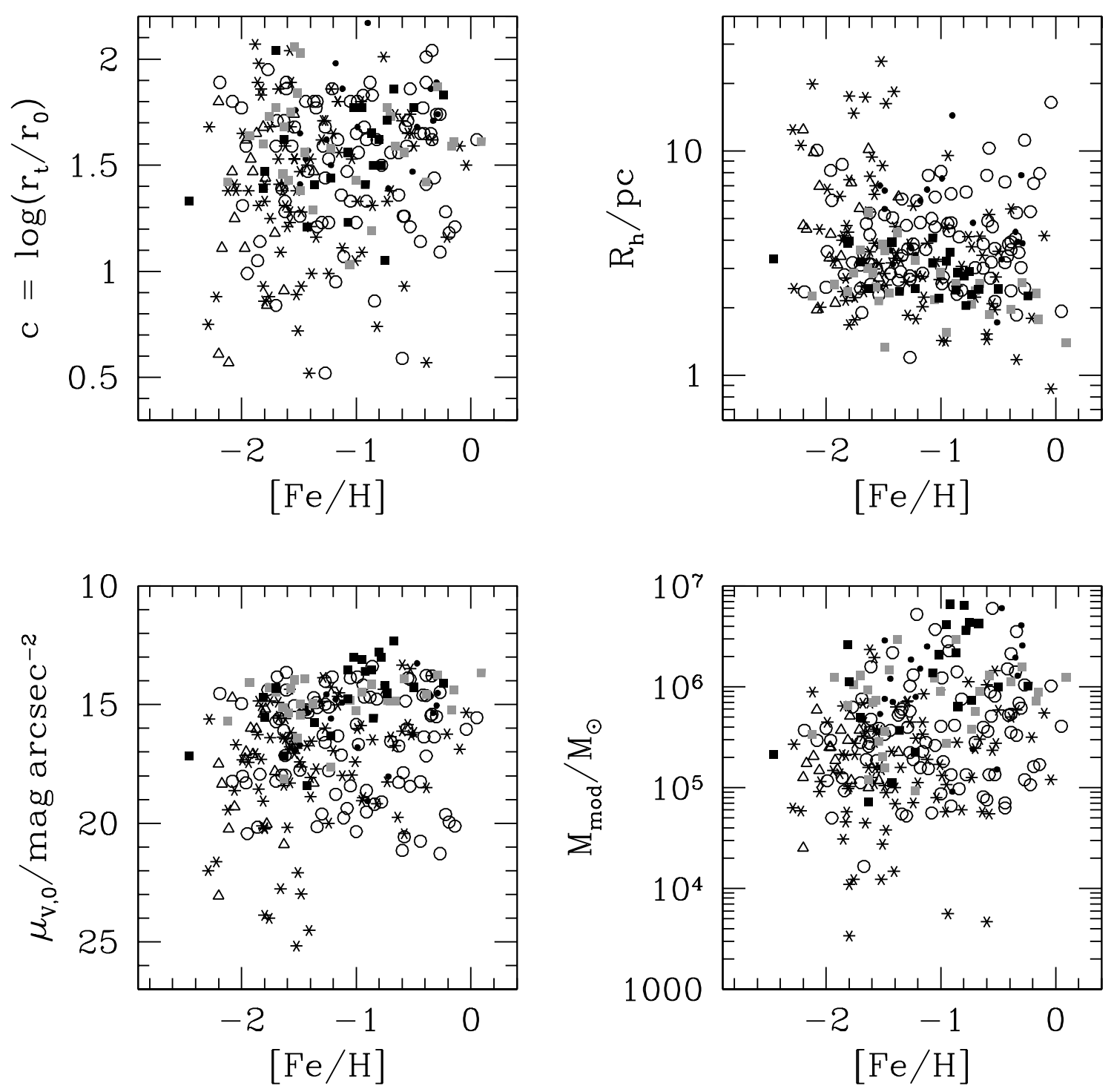

Fig. 11.- Structural parameters as a function of metallicity for globular clusters. Symbols as in Figure 10. Only clusters with measured values of $[\mathrm{Fe} / \mathrm{H}]$ (not assumed average values) are shown. 

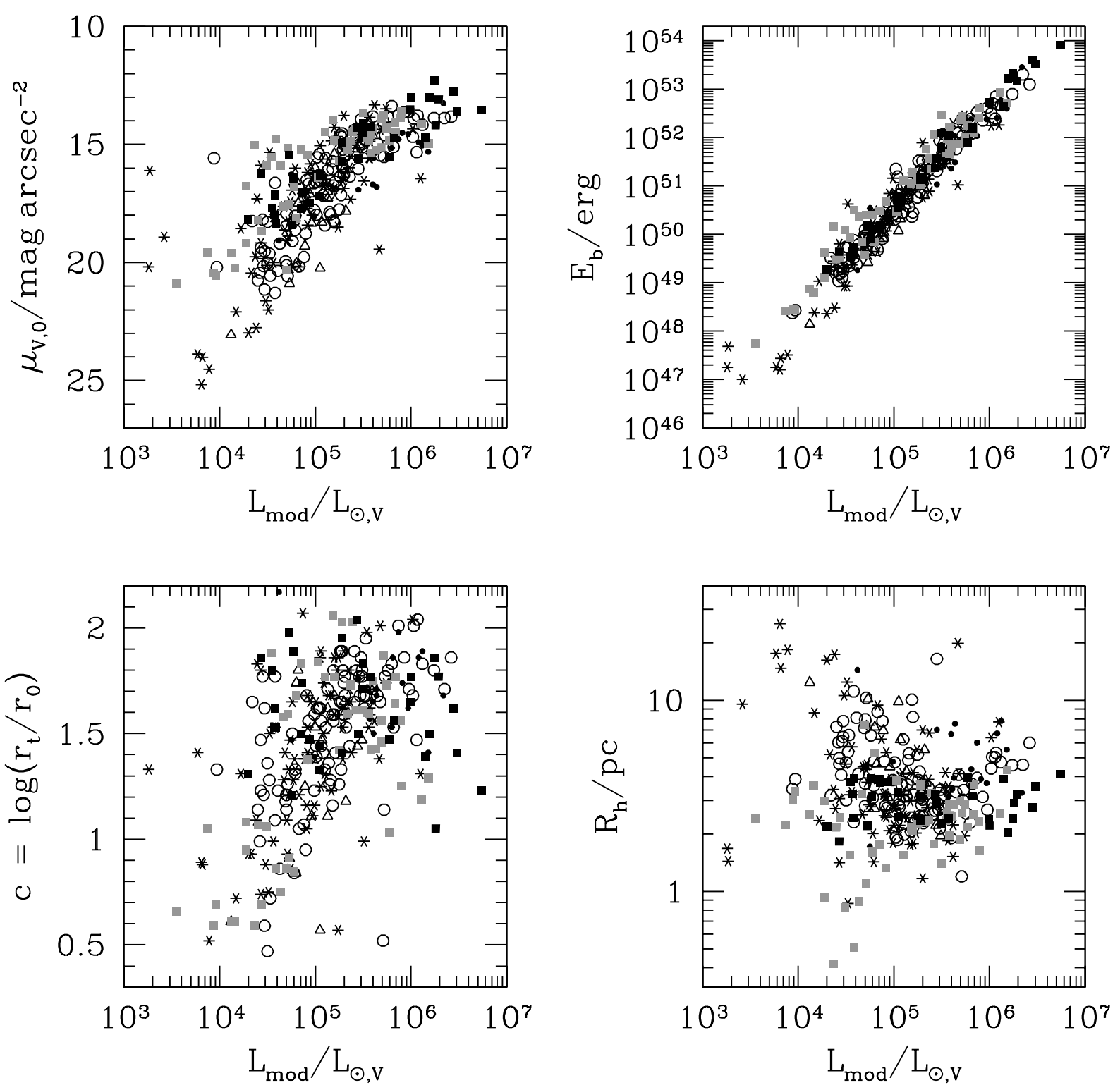

Fig. 12.- Structural parameters as a function of luminosity for globular clusters. Symbols as in Figure 10. 


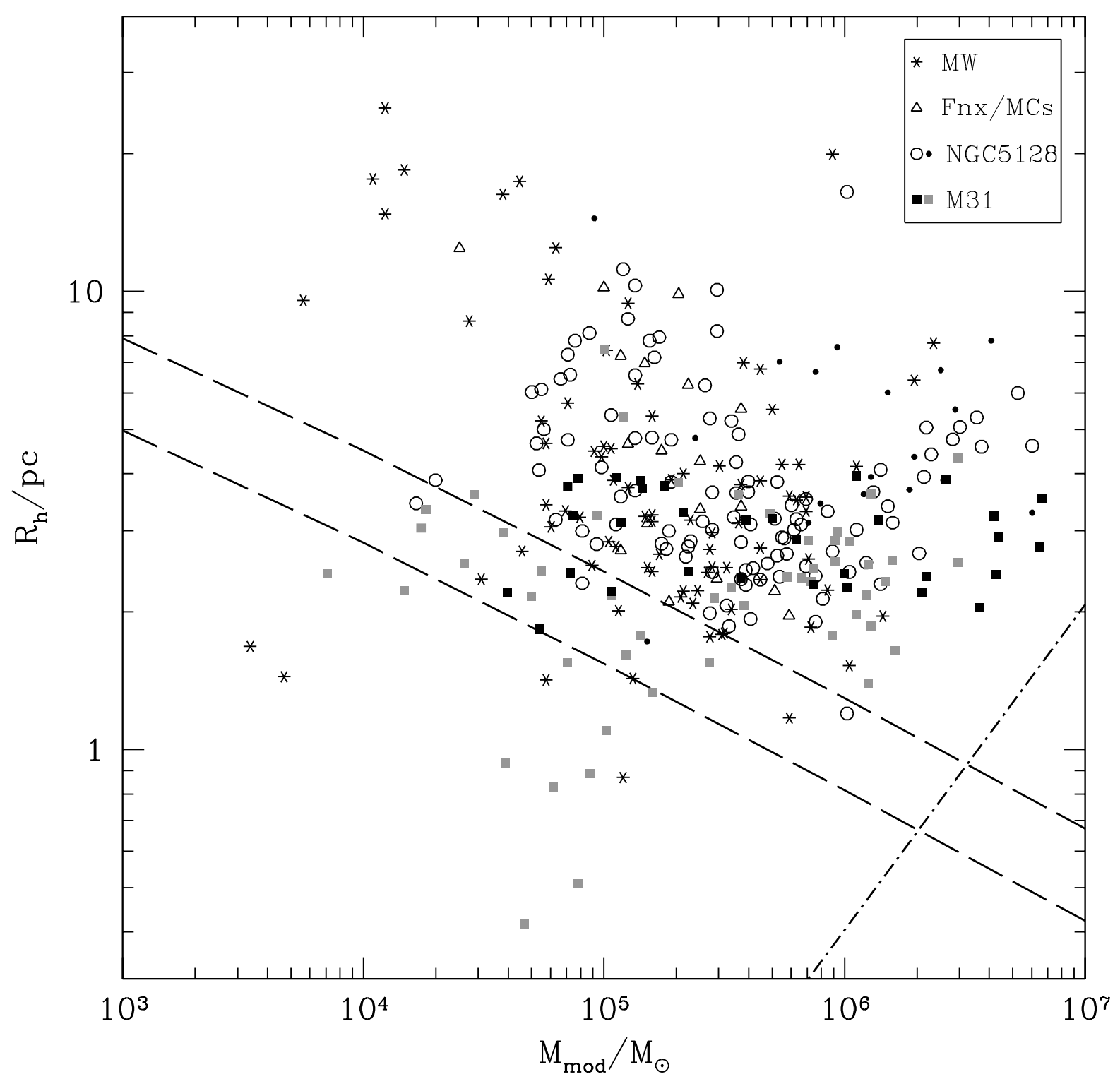

Fig. 13.- Projected half-light radius as a function of cluster mass for GCs in the Milky Way, Magellanic Clouds and Fornax, M31, and NGC 5128. Symbols: Milky Way (stars), the Magellanic Clouds and Fornax dwarf spheroidal (open triangles), NGC 5128 [open circles are clusters from McLaughlin et al. 2007a; small filled circles are from Harris et al. 2002], and M31 (filled squares). The darker filled squares are the M31 clusters observed with ACS or STIS; lighter squares are WFPC2 observations. Long-dashed lines are 'survival' lines; dot-dashed line is lower $R_{h}$ bound defined by McLaughlin et al. (2007b). 


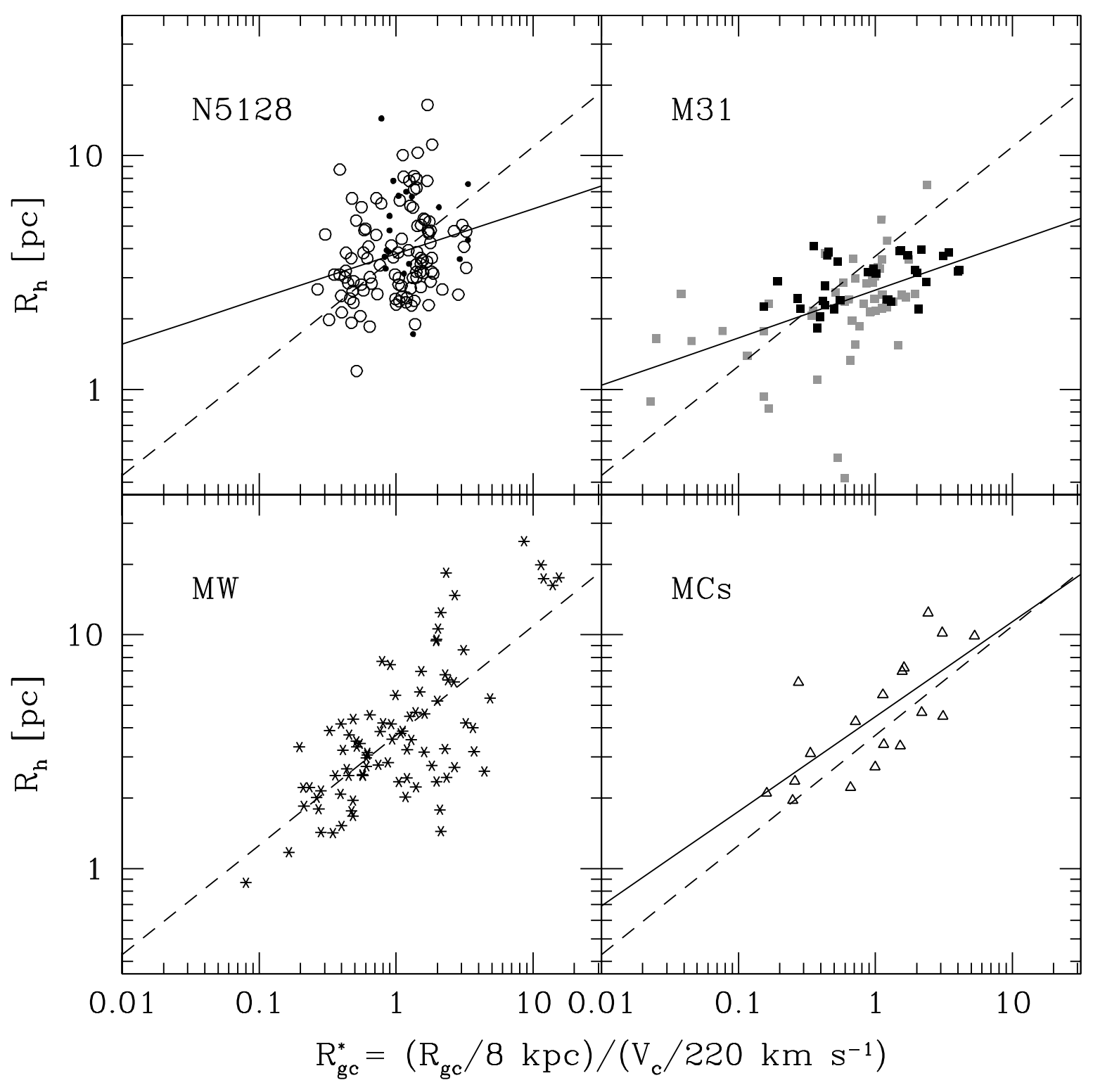

Fig. 14.- Projected half-light radius as a function of re-normalized galactocentric distance $R_{\mathrm{gc}}^{*}$ for globular clusters in the Milky Way (lower left), Magellanic Clouds and Fornax (lower right), M31 (upper right), and NGC 5128 (upper left). Solid lines are least-squares fits; dashed line in each panel is the Milky Way least-squares fit. 


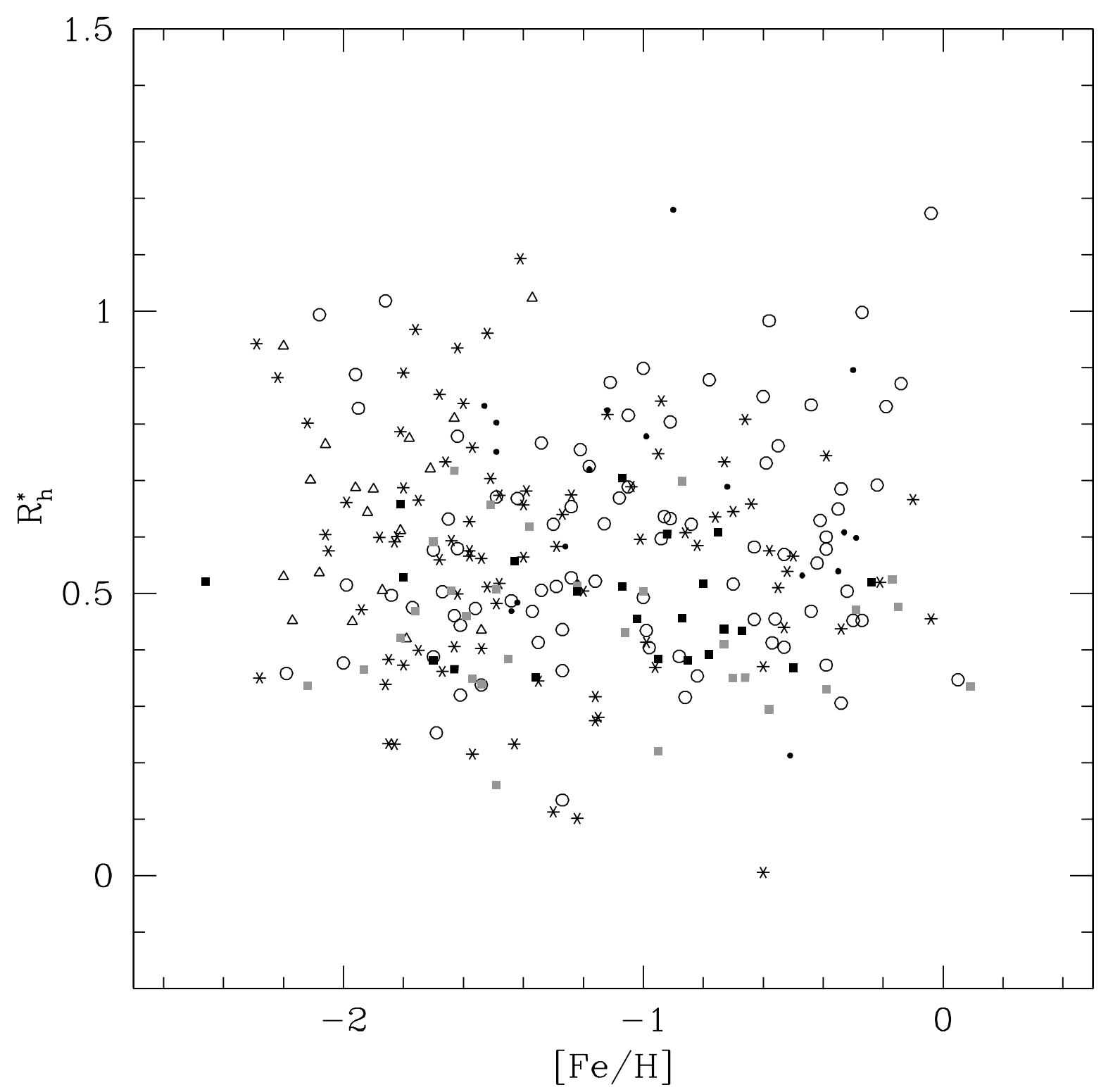

Fig. 15.- Re-normalized half-light radius $R_{h}^{*}$ as a function of metallicity for globular clusters in the Milky Way, Magellanic Clouds and Fornax, M31, and NGC 5128. Symbols as in Figure 13, Only clusters with measured values of $[\mathrm{Fe} / \mathrm{H}]$ (not assumed average values) are shown. 


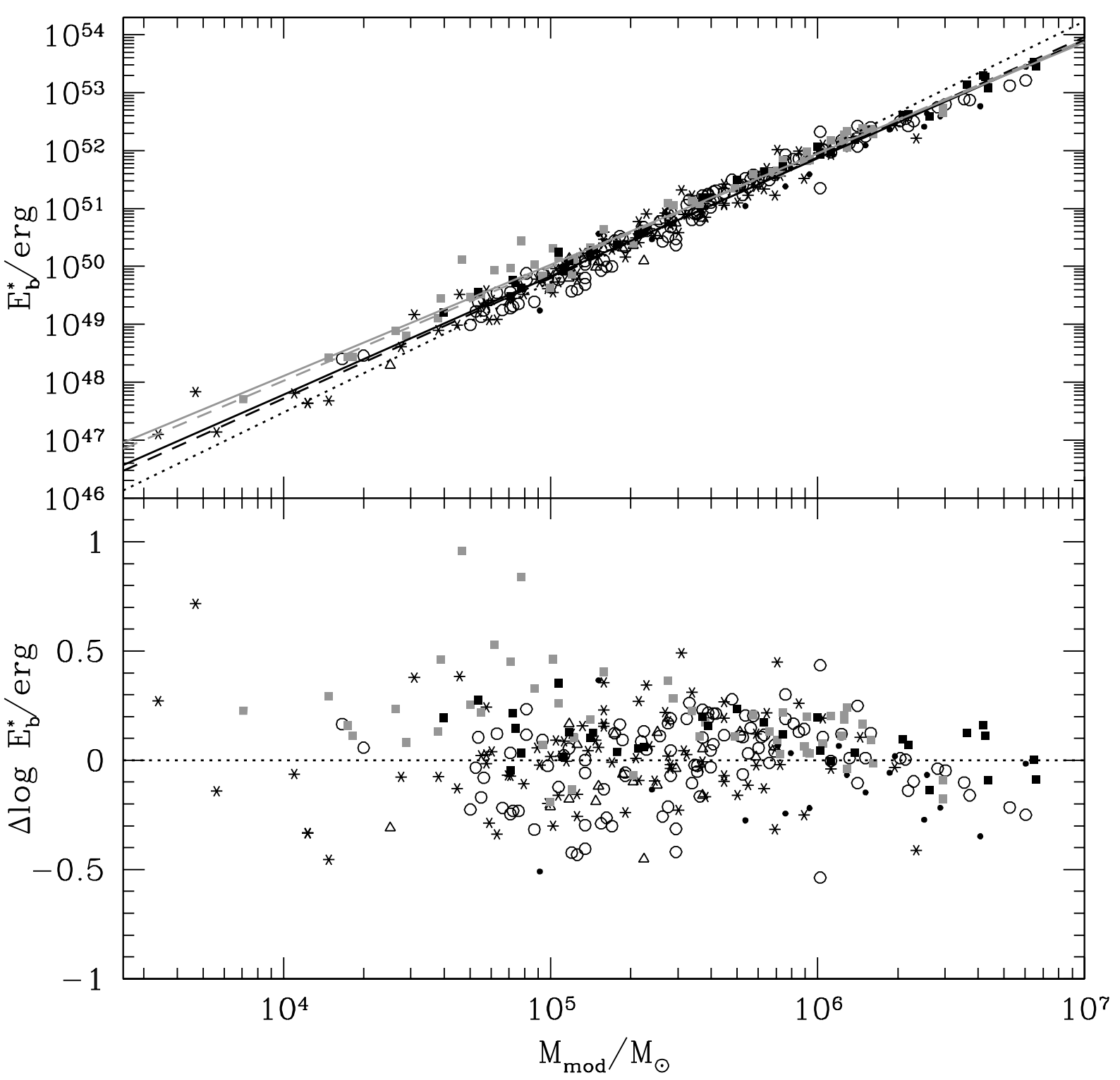

Fig. 16. - Re-normalized binding energy $E_{b}^{*}$ (top) and difference from Milky Way leastsquares fit (bottom), as a function of mass for globular clusters in the Milky Way, M31, and NGC 5128. Symbols as in Figure 13; lines in top panel are least-squares fits for Milky Way (black solid line), all M31 clusters (grey solid line), M31 ACS/STIS clusters only (grey dashed line), NGC 5128 clusters (black dashed line), and Magellanic Cloud and Fornax clusters (dotted line). 
Table 1. Integrated measurements for M31 clusters

\begin{tabular}{|c|c|c|c|c|c|c|c|c|}
\hline Cluster & $\epsilon$ & $\begin{array}{c}\theta \\
{\left[{ }^{\circ} \mathrm{E} \text { of } \mathrm{N}\right]}\end{array}$ & $\begin{array}{c}V \text { or }^{2} \text { alt. }^{\mathrm{a}} \\
\text { [VEGAMAG] }^{2}\end{array}$ & $\begin{array}{c}F 814 W \\
\text { [VEGAMAG] }\end{array}$ & $E(B-V)$ & $\begin{array}{c}R_{\mathrm{gc}} \\
{[\mathrm{kpc}]}\end{array}$ & {$[\mathrm{Fe} / \mathrm{H}]$} & source $^{b}$ \\
\hline \multicolumn{9}{|c|}{ ACS/WFC targets from program 10260} \\
\hline B037 & 0.27 & 54 & 16.06 & 14.07 & 1.38 & 2.97 & $-1.07 \pm 0.2$ & $\mathrm{P} 02$ \\
\hline B042 & 0.10 & 134 & 15.71 & 14.29 & 0.77 & 3.31 & $-0.78 \pm 0.2$ & $\mathrm{P} 02$ \\
\hline B063 & 0.10 & 28 & 15.31 & 14.13 & 0.44 & 3.47 & $-0.87 \pm 0.3$ & HBK \\
\hline B082 & 0.05 & 161 & 15.02 & 13.63 & 0.72 & 3.61 & $-0.80 \pm 0.2$ & $\mathrm{P} 02$ \\
\hline B088 & 0.28 & 178 & 15.04 & 13.90 & 0.48 & 3.79 & $-1.81 \pm 0.1$ & $\mathrm{P} 02$ \\
\hline B151 & 0.07 & 8 & 13.26 & 13.26 & 0.38 & 1.61 & $-0.75 \pm 0.2$ & HBK \\
\hline \multicolumn{9}{|c|}{ Serendipitous clusters observed in program 10260} \\
\hline B056D & 0.22 & 117 & 18.81 & 17.72 & 0.08 & 4.20 & $-1.20 \pm 0.6$ & AVE \\
\hline B041 & 0.05 & 30 & 18.95 & 18.13 & 0.13 & 2.72 & $-1.22 \pm 0.2$ & $\mathrm{P} 02$ \\
\hline B061 & 0.14 & 2 & 16.83 & 15.62 & 0.37 & 3.58 & $-0.73 \pm 0.3$ & $\mathrm{P} 02$ \\
\hline B090 & 0.14 & 124 & 18.49 & 17.52 & 0.08 & 3.15 & $-1.20 \pm 0.6$ & AVE \\
\hline B147 & 0.10 & 172 & 16.03 & 15.04 & 0.08 & 1.29 & $-0.24 \pm 0.4$ & HBK \\
\hline $\mathrm{B} 162^{\mathrm{c}}$ & 0.30 & 47 & 17.62 & 16.54 & 0.31 & 2.27 & $-1.22 \pm 0.2$ & B00 \\
\hline \multicolumn{9}{|c|}{ ACS/HRC targets from programs 9719 and 9767} \\
\hline B023 & 0.13 & 60 & 13.88 & 12.72 & 0.36 & 4.45 & $-0.92 \pm 0.1$ & HBK \\
\hline B158 & 0.05 & 13 & 14.36 & 13.44 & 0.12 & 2.38 & $-1.02 \pm 0.0$ & $\mathrm{P} 02$ \\
\hline $\mathrm{B} 225$ & 0.05 & 3 & 13.87 & 12.89 & 0.15 & 4.65 & $-0.67 \pm 0.1$ & $\mathrm{P} 02$ \\
\hline G001 & 0.20 & 162 & 13.79 & $\ldots$ & 0.08 & 34.55 & $-0.95 \pm 0.1$ & MEY \\
\hline \multicolumn{9}{|c|}{ STIS targets from program 8640} \\
\hline B020 & 0.05 & 40 & 14.97 & $\cdots$ & 0.08 & 7.37 & $-1.07 \pm 0.1$ & HBK \\
\hline B023 & 0.12 & 81 & 14.12 & $\ldots$ & 0.36 & 4.45 & $-0.92 \pm 0.1$ & HBK \\
\hline B196 & 0.05 & 60 & 17.36 & $\cdots$ & 0.24 & 8.10 & $-2.46 \pm 0.6$ & B00 \\
\hline B236 & 0.16 & 149 & 17.44 & $\ldots$ & 0.08 & 8.49 & $-1.20 \pm 0.6$ & AVE \\
\hline B289 & 0.13 & 39 & 16.27 & $\ldots$ & 0.08 & 16.88 & $-1.20 \pm 0.6$ & AVE \\
\hline B336 & 0.00 & $\ldots$ & 17.91 & $\ldots$ & 0.08 & 12.91 & $-1.20 \pm 0.6$ & AVE \\
\hline B351 & 0.05 & 110 & 17.61 & $\cdots$ & 0.11 & 12.57 & $-1.43 \pm 0.2$ & B00 \\
\hline B361 & 0.16 & 0 & 17.08 & $\cdots$ & 0.08 & 14.43 & $-1.20 \pm 0.6$ & AVE \\
\hline B363 & 0.15 & 173 & 17.91 & $\ldots$ & 0.08 & 10.44 & $-1.63 \pm 0.4$ & B00 \\
\hline B373 & 0.09 & 22 & 15.63 & $\cdots$ & 0.10 & 10.07 & $-0.50 \pm 0.2$ & HBK \\
\hline B396 ${ }^{\mathrm{c}}$ & 0.18 & 152 & 17.45 & $\ldots$ & 0.08 & 17.27 & $-1.20 \pm 0.6$ & AVE \\
\hline B405 & 0.09 & 133 & 15.25 & $\ldots$ & 0.12 & 18.20 & $-1.80 \pm 0.3$ & HBK \\
\hline B407 & 0.06 & 173 & 16.11 & $\ldots$ & 0.16 & 19.76 & $-0.85 \pm 0.3$ & HBK \\
\hline B422 & 0.41 & 149 & 17.98 & $\ldots$ & 0.08 & 16.34 & $-1.20 \pm 0.6$ & AVE \\
\hline B461 & 0.16 & 105 & 17.44 & $\ldots$ & 0.47 & 10.87 & $-1.36 \pm 0.5$ & B00 \\
\hline B462 & 0.09 & 70 & 18.03 & $\cdots$ & 0.08 & 3.74 & $-1.20 \pm 0.6$ & AVE \\
\hline G002 & 0.09 & 106 & 15.93 & $\ldots$ & 0.08 & 33.62 & $-1.70 \pm 0.4$ & HBK \\
\hline G339 & 0.10 & 174 & 17.35 & $\ldots$ & 0.08 & 28.68 & $-1.20 \pm 0.6$ & AVE \\
\hline G353 & 0.16 & 121 & 17.31 & $\ldots$ & 0.08 & 26.32 & $-1.20 \pm 0.6$ & AVE \\
\hline \multicolumn{9}{|c|}{ WFPC2-observed clusters from Barmby et al. (2002) } \\
\hline B006 & 0.08 & 74 & 15.46 & $\cdots$ & 0.13 & 6.39 & $-0.58 \pm 0.12$ & HBK \\
\hline B011 & 0.09 & 76 & 16.58 & $\ldots$ & 0.08 & 7.68 & $-1.54 \pm 0.34$ & HBK \\
\hline $\mathrm{B} 012$ & 0.08 & 46 & 15.04 & $\ldots$ & 0.11 & 5.74 & $-1.70 \pm 0.22$ & $\mathrm{P} 02$ \\
\hline B018 & 0.15 & 4 & 17.53 & .. & 0.08 & 9.27 & $-1.63 \pm 0.77$ & $\mathrm{P} 02$ \\
\hline B027 & 0.07 & 97 & 15.56 & $\ldots$ & 0.16 & 5.99 & $-1.64 \pm 0.32$ & HBK \\
\hline
\end{tabular}


Table 1-Continued

\begin{tabular}{|c|c|c|c|c|c|c|c|c|}
\hline Cluster & $\epsilon$ & $\begin{array}{c}\theta \\
{\left[{ }^{\circ} \mathrm{E} \text { of } \mathrm{N}\right]}\end{array}$ & $\begin{array}{c}V \text { or }^{2} \text { alt. }^{\mathrm{a}} \\
\text { [VEGAMAG] }^{2}\end{array}$ & $\begin{array}{c}F 814 W \\
{[\text { VEGAMAG] }}\end{array}$ & $E(B-V)$ & $\begin{array}{c}R_{\mathrm{gc}} \\
{[\mathrm{kpc}]}\end{array}$ & {$[\mathrm{Fe} / \mathrm{H}]$} & source $^{\mathrm{b}}$ \\
\hline B030 & 0.10 & 118 & 17.38 & $\ldots$ & 0.66 & 5.64 & $-0.39 \pm 0.36$ & P02 \\
\hline B045 & 0.08 & 40 & 15.78 & $\cdots$ & 0.18 & 4.87 & $-1.00 \pm 0.26$ & $\mathrm{P} 02$ \\
\hline B058 & 0.10 & 138 & 14.93 & $\ldots$ & 0.12 & 6.94 & $-1.45 \pm 0.24$ & HBK \\
\hline B068 & 0.22 & 42 & 16.41 & $\cdots$ & 0.45 & 4.31 & $-0.29 \pm 0.59$ & HBK \\
\hline B076 & 0.09 & 69 & 16.93 & $\ldots$ & 0.21 & 2.85 & $-0.73 \pm 0.07$ & $\mathrm{P} 02$ \\
\hline B109 & 0.10 & 72 & 16.20 & $\ldots$ & 0.08 & 1.39 & $-0.17 \pm 0.49$ & P02 \\
\hline B110 & 0.05 & 49 & 15.36 & $\ldots$ & 0.16 & 2.92 & $-1.06 \pm 0.14$ & P02 \\
\hline B114 & 0.06 & 132 & 17.44 & $\ldots$ & 0.16 & 0.88 & $-1.20 \pm 0.60$ & AVE \\
\hline B115 & 0.08 & 63 & 16.00 & $\ldots$ & 0.08 & 0.64 & $-0.15 \pm 0.38$ & HBK \\
\hline B123 & 0.14 & 62 & 17.42 & $\ldots$ & 0.08 & 1.28 & $-1.20 \pm 0.60$ & AVE \\
\hline B124 & 0.07 & 164 & 14.78 & $\cdots$ & 0.08 & 0.21 & $-1.20 \pm 0.60$ & AVE \\
\hline B127 & 0.08 & 64 & 14.47 & $\ldots$ & 0.15 & 0.32 & $-0.87 \pm 0.17$ & P02 \\
\hline B143 & 0.05 & 158 & 15.95 & $\ldots$ & 0.13 & 0.97 & $0.09 \pm 0.42$ & HBK \\
\hline B155 & 0.12 & 80 & 18.01 & $\ldots$ & 0.16 & 3.14 & $-1.20 \pm 0.60$ & AVE \\
\hline B156 & 0.05 & 67 & 16.97 & $\cdots$ & 0.08 & 3.60 & $-1.51 \pm 0.38$ & P02 \\
\hline B160 & 0.18 & 2 & 18.08 & $\cdots$ & 0.08 & 3.56 & $-1.17 \pm 1.25$ & P02 \\
\hline B231 & 0.17 & 136 & 17.25 & $\ldots$ & 0.08 & 5.54 & $-1.49 \pm 0.41$ & $\mathrm{P} 02$ \\
\hline B232 & 0.18 & 42 & 15.65 & $\cdots$ & 0.07 & 4.96 & $-1.81 \pm 0.17$ & $\mathrm{P} 02$ \\
\hline B233 & 0.11 & 74 & 15.72 & $\ldots$ & 0.12 & 8.05 & $-1.59 \pm 0.32$ & HBK \\
\hline B234 & 0.07 & 71 & 16.78 & $\cdots$ & 0.08 & 6.00 & $-0.95 \pm 0.13$ & P02 \\
\hline B240 & 0.16 & 98 & 15.18 & $\ldots$ & 0.08 & 7.22 & $-1.76 \pm 0.18$ & HBK \\
\hline B264 & 0.26 & 142 & 17.58 & $\cdots$ & 0.08 & 0.38 & $-1.20 \pm 0.60$ & AVE \\
\hline B268 & 0.12 & 103 & 18.31 & $\ldots$ & 0.08 & 1.39 & $-1.20 \pm 0.60$ & AVE \\
\hline B279 & 0.20 & 79 & 18.55 & $\cdots$ & 0.08 & 7.74 & $-1.20 \pm 0.60$ & AVE \\
\hline B311 & 0.09 & 54 & 15.45 & $\ldots$ & 0.23 & 13.06 & $-1.93 \pm 0.08$ & P02 \\
\hline B315 & 0.13 & 159 & 16.47 & $\cdots$ & 0.08 & 12.62 & $-2.11 \pm 0.53$ & HBK \\
\hline B317 & 0.11 & 66 & 16.57 & $\cdots$ & 0.13 & 10.14 & $-2.12 \pm 0.36$ & HBK \\
\hline B319 & 0.0 & 0 & 17.61 & $\ldots$ & 0.08 & 11.80 & $-2.27 \pm 0.47$ & B00 \\
\hline B324 & 0.0 & 0 & 18.45 & $\cdots$ & 0.08 & 8.29 & $-1.20 \pm 0.60$ & AVE \\
\hline B328 & 0.27 & 159 & 17.86 & $\ldots$ & 0.08 & 8.09 & $-1.22 \pm 0.80$ & HBK \\
\hline B330 & 0.14 & 102 & 17.72 & $\cdots$ & 0.08 & 8.46 & $-1.20 \pm 0.60$ & AVE \\
\hline B331 & 0.24 & 70 & 18.19 & $\ldots$ & 0.08 & 8.31 & $-1.44 \pm 0.69$ & B00 \\
\hline B333 & 0.23 & 26 & 18.84 & $\cdots$ & 0.08 & 7.95 & $-1.20 \pm 0.60$ & AVE \\
\hline B338 & 0.06 & 102 & 14.20 & $\ldots$ & 0.12 & 10.21 & $-1.38 \pm 0.09$ & HBK \\
\hline B343 & 0.09 & 70 & 16.31 & $\cdots$ & 0.07 & 14.71 & $-1.49 \pm 0.17$ & HBK \\
\hline B358 & 0.12 & 63 & 15.22 & $\ldots$ & 0.08 & 19.78 & $-1.83 \pm 0.22$ & HBK \\
\hline B368 & 0.0 & 0 & 17.92 & $\ldots$ & 0.08 & 9.52 & $-1.20 \pm 0.60$ & AVE \\
\hline B374 & 0.21 & 106 & 18.31 & $\cdots$ & 0.08 & 9.64 & $-1.90 \pm 0.67$ & $\mathrm{P} 02$ \\
\hline B379 & 0.09 & 55 & 16.18 & $\ldots$ & 0.10 & 11.30 & $-0.70 \pm 0.35$ & HBK \\
\hline B384 & 0.20 & 121 & 15.75 & $\cdots$ & 0.12 & 16.36 & $-0.66 \pm 0.22$ & HBK \\
\hline B386 & 0.08 & 140 & 15.55 & $\cdots$ & 0.08 & 14.02 & $-1.57 \pm 0.17$ & P02 \\
\hline B468 & 0.0 & 0 & 17.79 & $\cdots$ & 0.08 & 20.05 & $-1.20 \pm 0.60$ & AVE \\
\hline ВH04 & 0.32 & 128 & 19.69 & $\ldots$ & 0.08 & 8.33 & $-1.20 \pm 0.60$ & AVE \\
\hline BH05 & 0.19 & 123 & 16.06 & $\ldots$ & 0.08 & 9.01 & $-1.20 \pm 0.60$ & AVE \\
\hline BH11 & 0 & 0 & 19.86 & $\ldots$ & 0.08 & 9.41 & $-1.20 \pm 0.60$ & AVE \\
\hline
\end{tabular}


Table 1-Continued

\begin{tabular}{lrrrrrrrr}
\hline \hline Cluster & $\epsilon$ & $\begin{array}{c}\theta \\
{\left[{ }^{\circ} \mathrm{E} \text { of N] }\right.}\end{array}$ & $\begin{array}{c}V \text { or alt. }^{\mathrm{a}} \\
\text { [VEGAMAG] }^{2}\end{array}$ & $\begin{array}{c}F 814 W \\
\text { [VEGAMAG] }\end{array}$ & $E(B-V)$ & $\begin{array}{c}R_{\mathrm{gc}} \\
{[\mathrm{kpc}]}\end{array}$ & {$[\mathrm{Fe} / \mathrm{H}]$} & source $^{\mathrm{b}}$ \\
\hline BH18 & 0.10 & 116 & 18.18 & $\ldots$ & 0.08 & 12.35 & $-1.20 \pm 0.60$ & AVE \\
BH20 & 0.18 & 175 & 18.07 & $\ldots$ & 0.08 & 4.44 & $-1.20 \pm 0.60$ & AVE \\
BH21 & 0.15 & 30 & 18.61 & $\ldots$ & 0.08 & 5.01 & $-1.20 \pm 0.60$ & AVE \\
BH23 & 0.09 & 75 & 18.83 & $\ldots$ & 0.08 & 1.28 & $-1.20 \pm 0.60$ & AVE \\
BH24 & 0.38 & 44 & 20.67 & $\ldots$ & 0.08 & 5.37 & $-1.20 \pm 0.60$ & AVE \\
BH29 & 0 & 0 & 19.65 & $\ldots$ & 0.08 & 8.71 & $-1.20 \pm 0.60$ & AVE \\
DAO38 & 0.34 & 108 & 19.25 & $\ldots$ & 0.08 & 9.43 & $-1.20 \pm 0.60$ & AVE \\
M091 & 0 & 0 & 19.14 & $\ldots$ & 0.08 & 9.31 & $-1.20 \pm 0.60$ & AVE \\
NB39 & 0.13 & 28 & 17.94 & $\ldots$ & 0.08 & 0.19 & $-1.20 \pm 0.60$ & AVE \\
\hline
\end{tabular}

${ }^{\mathrm{a}} \mathrm{F} 606 \mathrm{~W}$ for ACS-observed clusters, except G001 (F555W).

${ }^{\mathrm{b}} \mathrm{HBK}=$ Huchra et al. (1991); P02= Perrett et al. (2002); B00= Barmby et al. (2000); AVE=no measurement; $[\mathrm{Fe} / \mathrm{H}]=-1.2 \pm 0.6$ assigned.

${ }^{\mathrm{c}}$ These clusters appear close to the image edge; their measurements do not cover the full range of radial distance. 
Table 2. 49 F606W, F814W, or V Intensity Profiles for 33 GCs in M31

\begin{tabular}{ccccccc}
\hline \hline Name & Detector & Filter & $\begin{array}{c}R \\
{[\operatorname{arcsec}]} \\
(4)\end{array}$ & $\begin{array}{c}I \\
{\left[L_{\odot} \mathrm{pc}^{-2}\right]} \\
(5)\end{array}$ & $\begin{array}{c}\text { uncertainty } \\
{\left[L_{\odot} \mathrm{pc}^{-2}\right]}\end{array}$ & Flag \\
\hline B056D & WFC & F814W & 0.0260 & 2233.332 & 101.584 & OK \\
B056D & WFC & F814W & 0.0287 & 2232.822 & 107.622 & DEP \\
B056D & WFC & F814W & 0.0315 & 2233.302 & 112.895 & DEP \\
B056D & WFC & F814W & 0.0347 & 2236.559 & 117.452 & DEP \\
B056D & WFC & F814W & 0.0381 & 2241.757 & 122.118 & DEP \\
B056D & WFC & F814W & 0.0420 & 2245.391 & 127.393 & DEP \\
B056D & WFC & F814W & 0.0461 & 2245.646 & 133.215 & DEP \\
B056D & WFC & F814W & 0.0508 & 2239.541 & 138.504 & DEP \\
B056D & WFC & F814W & 0.0558 & 2234.688 & 143.454 & OK \\
B056D & WFC & F814W & 0.0614 & 2229.836 & 148.906 & DEP \\
B056D & WFC & F814W & 0.0676 & 2227.591 & 154.365 & DEP \\
\hline
\end{tabular}

Note. - Table 2 is published in its entirety in the electronic edition of the Journal. Only a small portion is shown here, for guidance regarding its form and content. See text for description of the FLAG column.

Table 3. PSF Models: $I_{\mathrm{PSF}}=I_{0}\left[1+\left(R / r_{0}\right)^{\alpha}\right]^{-\beta / \alpha}$

\begin{tabular}{llccccc}
\hline \hline Detector & \multirow{2}{*}{ Filter } & $r_{0}$ & $\alpha$ & $\beta$ & \multicolumn{2}{c}{ FWHM } \\
{$[\operatorname{arcsec}]$} & & & {$[\operatorname{arcsec}]$} & {$[\mathrm{px}]$} \\
\hline WFC & F606W & 0.0686 & 3 & 3.69 & 0.125 & 2.5 \\
WFC & F814W & 0.0783 & 3 & 3.56 & 0.145 & 2.9 \\
& & & & & & \\
HRC & F555W & 0.0267 & 3 & 3.09 & 0.0527 & 2.1 \\
HRC & F606W & 0.0294 & 3 & 2.95 & 0.0593 & 2.4 \\
HRC & F814W & 0.0351 & 3 & 2.96 & 0.0706 & 2.8 \\
STIS & CL & 0.074 & 2 & 4.2 & 0.093 & 1.8 \\
\hline
\end{tabular}


Table 4. Basic Parameters of Fits to 49 Profiles of 33 GCs in M31

\begin{tabular}{|c|c|c|c|c|c|c|c|c|c|c|c|c|}
\hline $\begin{array}{c}\text { Name } \\
(1)\end{array}$ & $\begin{array}{c}\text { Detector } \\
\text { (2) }\end{array}$ & $\begin{array}{l}\text { Ext. } \\
{[\mathrm{mag}]} \\
(3)\end{array}$ & $\begin{array}{c}V \text { color } \\
{[\mathrm{mag}]} \\
(4)\end{array}$ & $\begin{array}{l}N_{\mathrm{pts}} \\
(5)\end{array}$ & $\begin{array}{l}\text { Model } \\
\text { (6) }\end{array}$ & $\begin{array}{c}\chi_{\min }^{2} \\
(7)\end{array}$ & 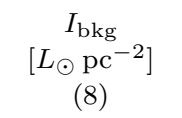 & $\begin{array}{l}W_{0} \\
(9)\end{array}$ & $\begin{array}{l}c / n \\
(10)\end{array}$ & $\begin{array}{c}\mu_{0} \\
{\left[\mathrm{mag} \operatorname{arcsec}^{-2}\right]} \\
(11)\end{array}$ & $\begin{array}{c}\log r_{0} \\
{[\operatorname{arcsec}]} \\
(12)\end{array}$ & $\begin{array}{c}\log r_{0} \\
{[\mathrm{pc}]} \\
(13)\end{array}$ \\
\hline B056D & WFC/F606 & 0.224 & $0.274 \pm 0.051$ & 52 & $\begin{array}{r}\text { K66 } \\
\text { W } \\
\text { S }\end{array}$ & $\begin{array}{l}149.50 \\
131.14 \\
195.14\end{array}$ & $\begin{array}{l}20.38 \pm 0.76 \\
19.98 \pm 0.97 \\
20.92 \pm 0.60\end{array}$ & $\begin{array}{r}6.20_{-0.60}^{+0.60} \\
6.10_{-0.70}^{+0.70} \\
\end{array}$ & $\begin{array}{l}1.31_{-0.15}^{+0.16} \\
1.84_{-0.28}^{+0.40} \\
1.26_{-0.20}^{+0.24}\end{array}$ & $\begin{array}{l}17.92_{-0.06}^{+0.05} \\
17.95_{-0.05}^{+0.05} \\
17.45_{-0.28}^{+0.23}\end{array}$ & $\begin{array}{l}-0.562_{-0.042}^{+0.042} \\
-0.526_{-0.043}^{+0.044} \\
-0.896_{-0.208}^{+0.161}\end{array}$ & $\begin{array}{r}0.016_{-0.042}^{+0.042} \\
0.051_{-0.043}^{+0.044} \\
-0.318_{-0.208}^{+0.161}\end{array}$ \\
\hline B056D & WFC/F814 & 0.144 & $1.286 \pm 0.064$ & 52 & $\begin{array}{r}\text { K66 } \\
\text { W } \\
\text { S }\end{array}$ & $\begin{array}{l}175.21 \\
149.55 \\
224.33\end{array}$ & $\begin{array}{l}27.74 \pm 1.46 \\
27.25 \pm 1.55 \\
28.56 \pm 1.00\end{array}$ & $\begin{array}{r}5.80_{-0.90}^{+0.80} \\
5.50_{-1.10}^{+0.80} \\
-\end{array}$ & $\begin{array}{l}1.21_{-0.20}^{+0.21} \\
1.59_{-0.33}^{+0.35} \\
1.14_{-0.20}^{+0.28}\end{array}$ & $\begin{array}{l}16.88_{-0.08}^{+0.08} \\
16.91_{-0.06}^{+0.07} \\
16.51_{-0.35}^{+0.25}\end{array}$ & $\begin{array}{l}-0.561_{-0.068}^{+0.068} \\
-0.515_{-0.053}^{+0.075} \\
-0.825_{-0.1643}^{+0.161}\end{array}$ & $\begin{array}{r}0.016_{-0.061}^{+0.068} \\
0.063_{-0.053}^{+0.075} \\
-0.248_{-0.243}^{+0.161}\end{array}$ \\
\hline B090 & WFC/F814 & 0.144 & $1.114 \pm 0.064$ & 50 & $\begin{array}{r}\text { K66 } \\
\text { W } \\
\text { S }\end{array}$ & $\begin{array}{l}105.21 \\
107.84 \\
124.24\end{array}$ & $\begin{array}{l}76.95 \pm 2.49 \\
75.18 \pm 4.06 \\
77.42 \pm 2.29\end{array}$ & $\begin{array}{r}7.50_{-0.50}^{+0.60} \\
7.50_{-0.70}^{+0.70} \\
-\end{array}$ & $\begin{array}{l}1.68_{-0.15}^{+0.18} \\
2.79_{-0.55}^{+0.45} \\
2.30_{-0.44}^{+0.60}\end{array}$ & $\begin{array}{l}15.71_{-0.11}^{+0.09} \\
15.76_{-0.09}^{+0.09} \\
14.10_{-0.81}^{+0.60}\end{array}$ & $\begin{array}{r}-0.898_{-0.055}^{+0.045} \\
-0.860_{-0.052}^{+0.053} \\
-2.135_{-0.684}^{+0.465}\end{array}$ & $\begin{array}{r}-0.320_{-0.058}^{+0.045} \\
-0.282_{-0.052}^{+0.052} \\
-1.557_{-0.468}^{+0.465}\end{array}$ \\
\hline
\end{tabular}

Note. - See text for column descriptions. Table 4 is available in its entirety in the electronic edition of the Journal. A short extract from it is shown here, for guidance regarding its form and content. 
Table 5. Derived Structural and Photometric Parameters of GCs in M31

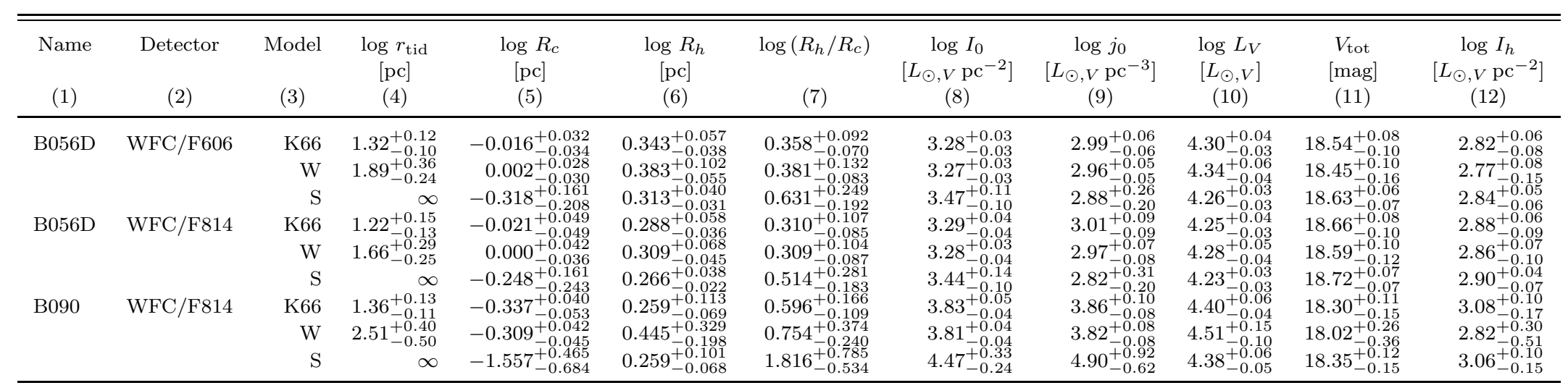

Note. - See text for column descriptions. Table 5 is available in its entirety in the electronic edition of the Journal. A short extract from it is shown here, for guidance regarding its form and content. 
Table 6. Derived Dynamical Parameters of GCs in M31

\begin{tabular}{|c|c|c|c|c|c|c|c|c|c|c|c|c|}
\hline $\begin{array}{l}\text { Name } \\
(1)\end{array}$ & $\begin{array}{c}\text { Detector } \\
\text { (2) }\end{array}$ & $\begin{array}{c}\Upsilon_{V}^{\text {pop }} \\
{\left[M_{\odot} L_{\odot, V}^{-1}\right]} \\
(3)\end{array}$ & $\begin{array}{l}\text { Model } \\
(4)\end{array}$ & $\begin{array}{c}\log M_{\text {tot }} \\
{\left[M_{\odot}\right]} \\
(5)\end{array}$ & $\begin{array}{c}\log E_{b} \\
{[\mathrm{erg}]} \\
(6)\end{array}$ & $\begin{array}{c}\log \Sigma_{0} \\
{\left[M_{\odot} \mathrm{pc}^{-2}\right]} \\
(7)\end{array}$ & $\begin{array}{c}\log \rho_{0} \\
{\left[M_{\odot} \mathrm{pc}^{-3}\right]} \\
(8)\end{array}$ & $\begin{array}{c}\log \Sigma_{\mathrm{h}} \\
{\left[M_{\odot} \mathrm{pc}^{-2}\right]} \\
(9)\end{array}$ & $\begin{array}{c}\log \sigma_{\mathrm{p}, 0} \\
{\left[\mathrm{~km} \mathrm{~s}^{-1}\right]} \\
\quad(10)\end{array}$ & $\begin{array}{c}\log v_{\text {esc }, 0} \\
{\left[\mathrm{~km} \mathrm{~s}^{-1}\right]} \\
(11)\end{array}$ & $\begin{array}{l}\log t_{\mathrm{rh}} \\
{[\mathrm{yr}]} \\
(12)\end{array}$ & $\begin{array}{c}\log f_{0} \\
\left.\left[M_{\odot}(\mathrm{pc} \mathrm{km} \mathrm{s})^{-1}\right)^{-3}\right] \\
(13)\end{array}$ \\
\hline B056D & WFC/F606 & $2.005_{-0.263}^{+0.617}$ & $\begin{array}{r}\text { K66 } \\
\text { W } \\
\text { S }\end{array}$ & $\begin{array}{l}4.60_{-0.07}^{+0.12} \\
4.64_{-0.07}^{+0.13} \\
4.56^{+0.12}\end{array}$ & $\begin{array}{l}49.27_{-0.09}^{+0.17} \\
49.29_{-0.09}^{+0.17} \\
49.24_{-0.00}^{+0.17}\end{array}$ & $\begin{array}{l}3.58_{-0.07}^{+0.12} \\
3.57_{-0.07}^{+0.012} \\
3.77_{-0.16}^{+0.16}\end{array}$ & $\begin{array}{l}3.29_{-0.08}^{+0.13} \\
3.26_{-0.08}^{+0.03} \\
3.18_{-0.27}^{+0.27}\end{array}$ & $\begin{array}{l}3.12_{-0.10}^{+0.13} \\
3.07_{-0.16}^{+0.14} \\
3.14_{-0.13}^{+0.13}\end{array}$ & $\begin{array}{l}0.526_{-0.033}^{+0.059} \\
0.530_{-0.033}^{+0.059} \\
0.518_{-0.036}^{+0.060}\end{array}$ & $\begin{array}{l}1.101_{-0.033}^{+0.059} \\
1.116_{-0.033}^{+0.059} \\
1.123_{-0.020}^{+0.059}\end{array}$ & $\begin{array}{l}8.43_{-0.07}^{+0.12} \\
8.51_{-0.10}^{+0.19} \\
8.37^{+0.09}\end{array}$ & $\begin{array}{l}0.484_{-0.087}^{+0.103} \\
0.434_{-0.097}^{+0.097} \\
0.433_{-0.37}^{+0.377}\end{array}$ \\
\hline B056D & WFC/F814 & $2.005_{-0.263}^{+0.617}$ & $\begin{array}{r}\text { K66 } \\
\text { W } \\
\text { S }\end{array}$ & $\begin{array}{l}4.56_{-0.07}^{+0.12} \\
4.58_{-0.07}^{+0.13} \\
4.53_{-0.07}^{+0.12}\end{array}$ & $\begin{array}{l}49.24_{-0.09}^{+0.17} \\
49.25_{-0.09}^{+0.17} \\
49.22_{-0.09}^{+0.17}\end{array}$ & $\begin{array}{l}3.60_{-0.07}^{+0.12} \\
3.58_{-0.07}^{+0.12} \\
3.74_{-0.12}^{+0.18}\end{array}$ & $\begin{array}{l}3.31_{-0.11}^{+0.15} \\
3.27_{-0.10}^{+0.13} \\
3.12_{-0.0}^{+0.31}\end{array}$ & $\begin{array}{l}3.18_{-0.11}^{+0.13} \\
3.17_{-0.12}^{+0.13} \\
3.20_{-0.09}^{+0.12}\end{array}$ & $\begin{array}{l}0.529_{-0.034}^{+0.060} \\
0.534_{-0.034}^{+0.060} \\
0.524_{-0.037}^{+0.060}\end{array}$ & $\begin{array}{l}1.095_{-0.035}^{+0.060} \\
1.110_{-0.034}^{+0.060} \\
1.121_{-0.034}^{+0.060}\end{array}$ & $\begin{array}{l}8.31_{-0.07}^{+0.12} \\
8.35_{-0.09}^{+0.13} \\
8.27_{-0.05}^{+0.09}\end{array}$ & $\begin{array}{l}0.485_{-0.127}^{+0.134} \\
0.423_{-0.113}^{+0.108} \\
0.324_{-0.252}^{+0.425}\end{array}$ \\
\hline B090 & WFC/F814 & $2.005_{-0.263}^{+0.617}$ & $\begin{array}{r}\text { K66 } \\
\text { W } \\
\text { S }\end{array}$ & $\begin{array}{l}4.70_{-0.08}^{+0.13} \\
4.81_{-0.12}^{+0.19} \\
4.68_{-0.08}^{+0.13}\end{array}$ & $\begin{array}{l}49.56_{-0.09}^{+0.17} \\
49.59_{-0.10}^{+0.17} \\
49.52_{-0.09}^{+0.17}\end{array}$ & $\begin{array}{l}4.13_{-0.07}^{+0.13} \\
4.12_{-0.07}^{+0.12} \\
4.78_{-0.25}^{+0.35}\end{array}$ & $\begin{array}{l}4.16_{-0.10}^{+0.15} \\
4.12_{-0.10}^{+0.14} \\
5.20_{-0.63}^{+0.92}\end{array}$ & $\begin{array}{l}3.38_{-0.18}^{+0.16} \\
3.12_{-0.52}^{+0.32} \\
3.36_{-0.16}^{+0.15}\end{array}$ & $\begin{array}{l}0.638_{-0.034}^{+0.060} \\
0.643_{-0.034}^{+0.060} \\
0.551_{-0.078}^{+0.074}\end{array}$ & $\begin{array}{l}1.240_{-0.034}^{+0.060} \\
1.254_{-0.034}^{+0.060} \\
1.274_{-0.036}^{+0.062}\end{array}$ & $\begin{array}{l}8.33_{-0.12}^{+0.20} \\
8.65_{-0.34}^{+0.56} \\
8.32_{-0.12}^{+0.18}\end{array}$ & $\begin{array}{l}1.031_{-0.096}^{+0.132} \\
0.962_{-0.104}^{+0.121} \\
2.707_{-0.929}^{+1.425}\end{array}$ \\
\hline
\end{tabular}

Note. - See text for column descriptions. Table 6 is available in its entirety in the electronic edition of the Journal. A short extract from it is shown here, for guidance |regarding its form and content. 
Table 7. Galactocentric Radii and $\kappa$-Space Parameters for GCs in M31

\begin{tabular}{cccccccc}
\hline \hline Name & Detector & $\begin{array}{c}R_{\mathrm{gc}} \\
{[\mathrm{kpc}]}\end{array}$ & Model & $\kappa_{m, 1}$ & $\kappa_{m, 2}$ & $\kappa_{m, 3}$ \\
$(1)$ & $(2)$ & $(3)$ & $(4)$ & $(5)$ & $(6)$ & $(7)$ \\
\hline \multirow{2}{*}{ B056D } & WFC/F606 & \multirow{2}{*}{4.20} & $\mathrm{~K} 66$ & $-1.136_{-0.049}^{+0.089}$ & $4.059_{-0.124}^{+0.158}$ & $0.341_{-0.002}^{+0.007}$ \\
& & & $\mathrm{~W}$ & $-1.101_{-0.055}^{+0.105}$ & $4.011_{-0.183}^{+0.169}$ & $0.347_{-0.005}^{+0.017}$ \\
& & & $\mathrm{~S}$ & $-1.168_{-0.047}^{+0.084}$ & $4.085_{-0.112}^{+0.156}$ & $0.336_{-0.007}^{+0.001}$ \\
B056D & WFC/F814 & \multirow{2}{*}{4.20} & $\mathrm{~K} 66$ & $-1.169_{-0.049}^{+0.089}$ & $4.136_{-0.130}^{+0.160}$ & $0.340_{-0.000}^{+0.005}$ \\
& & & $\mathrm{~W}$ & $-1.147_{-0.051}^{+0.093}$ & $4.120_{-0.138}^{+0.164}$ & $0.343_{-0.001}^{+0.008}$ \\
& & & $\mathrm{~S}$ & $-1.192_{-0.048}^{+0.084}$ & $4.156_{-0.152}^{+0.152}$ & $0.337_{-0.005}^{+0.000}$ \\
B090 & WFC/F814 & \multirow{2}{*}{3.15} & $\mathrm{~K} 66$ & $-1.036_{-0.064}^{+0.111}$ & $4.401_{-0.207}^{+0.185}$ & $0.366_{-0.014}^{+0.027}$ \\
& & & $\mathrm{~W}$ & $-0.898_{-0.141}^{+0.239}$ & $4.115_{-0.565}^{+0.358}$ & $0.415_{-0.051}^{+0.100}$ \\
& & & $\mathrm{~S}$ & $-1.159_{-0.055}^{+0.085}$ & $4.315_{-0.235}^{+0.205}$ & $0.277_{-0.054}^{+0.033}$ \\
\hline
\end{tabular}

Note. - See text for column descriptions, particularly difference between $\kappa$ definitions used here and those of Bender et al. (1992). Table 7 is available in its entirety in the electronic edition of the Journal. A short extract from it is shown here, for guidance regarding its form and content. 
Table 8. Predicted Aperture Velocity Dispersions for GCs in M31

\begin{tabular}{|c|c|c|c|c|c|c|c|c|c|c|}
\hline $\begin{array}{l}\text { Name } \\
(1)\end{array}$ & $\begin{array}{l}\text { Detector } \\
\qquad(2)\end{array}$ & $\begin{array}{c}\Upsilon_{V}^{\text {pop }} \\
{\left[M_{\odot} L_{\odot, V}^{-1}\right]} \\
(3)\end{array}$ & $\begin{array}{l}\text { Model } \\
\text { (4) }\end{array}$ & $\begin{array}{c}\log R_{h} \\
{[\mathrm{pc}]} \\
(5)\end{array}$ & $\begin{array}{c}\log R_{h} \\
{[\operatorname{arcsec}]} \\
\quad(6)\end{array}$ & $\begin{array}{c}\log \sigma_{\mathrm{ap}}\left(R_{h} / 8\right) \\
{\left[\mathrm{km} \mathrm{s}^{-1}\right]} \\
(7)\end{array}$ & $\begin{array}{c}\log \sigma_{\mathrm{ap}}\left(R_{h} / 4\right) \\
{\left[\mathrm{km} \mathrm{s}^{-1}\right]} \\
(8)\end{array}$ & $\begin{array}{c}\log \sigma_{\mathrm{ap}}\left(R_{h}\right) \\
{\left[\mathrm{km} \mathrm{s}^{-1}\right]} \\
(9)\end{array}$ & $\begin{array}{c}\log \sigma_{\mathrm{ap}}\left(4 R_{h}\right) \\
{\left[\mathrm{km} \mathrm{s}^{-1}\right]} \\
(10)\end{array}$ & $\begin{array}{c}\log \sigma_{\mathrm{ap}}\left(8 R_{h}\right) \\
{\left[\mathrm{km} \mathrm{s}^{-1}\right]} \\
(11)\end{array}$ \\
\hline B056D & WFC/F606 & $2.005_{-0.263}^{+0.617}$ & $\begin{array}{r}\text { K66 } \\
\text { W } \\
\text { S }\end{array}$ & $\begin{array}{l}0.343 \\
0.383 \\
0.313\end{array}$ & $\begin{array}{l}-0.235_{-0.038}^{+0.057} \\
-0.194_{-0.055}^{+0.102} \\
-0.265^{+0.040}\end{array}$ & $\begin{array}{l}0.525_{-0.033}^{+0.059} \\
0.529_{-0.033}^{+0.059} \\
0.494_{-0.022}^{+0.060}\end{array}$ & $\begin{array}{l}0.523_{-0.033}^{+0.059} \\
0.526_{-0.033}^{+0.059} \\
0.508_{-0.022}^{+0.060}\end{array}$ & $\begin{array}{l}0.501_{-0.033}^{+0.059} \\
0.499_{-0.064}^{+0.060} \\
0.503^{+0.060}\end{array}$ & $\begin{array}{l}0.454_{-0.033}^{+0.060} \\
0.453_{-0.034}^{+0.060} \\
0.454_{-0.024}^{+0.060}\end{array}$ & $\begin{array}{l}0.446_{-0.034}^{+0.060} \\
0.441_{-0.060}^{+0.060} \\
0.450_{-0.034}^{+0.060}\end{array}$ \\
\hline B056D & $\mathrm{WFC} / \mathrm{F} 814$ & $2.005_{-0.263}^{+0.617}$ & $\begin{array}{r}\text { K66 } \\
\text { W } \\
\text { S }\end{array}$ & $\begin{array}{l}0.288 \\
0.309 \\
0.266\end{array}$ & $\begin{array}{l}-0.289_{-0.036}^{+0.058} \\
-0.269_{-0.045}^{+0.068} \\
-0.311_{-0.022}^{+0.038}\end{array}$ & $\begin{array}{l}0.528_{-0.034}^{+0.060} \\
0.533_{-0.034}^{+0.060} \\
0.501_{-0.036}^{+0.061}\end{array}$ & $\begin{array}{l}0.527_{-0.035}^{+0.060} \\
0.531_{-0.034}^{+0.060} \\
0.514_{-0.035}^{+0.061}\end{array}$ & $\begin{array}{l}0.505_{-0.035}^{+0.060} \\
0.506_{-0.035}^{+0.060} \\
0.508_{-0.035}^{+0.060}\end{array}$ & $\begin{array}{l}0.458_{-0.035}^{+0.060} \\
0.460_{-0.035}^{+0.060} \\
0.459_{-0.035}^{+0.060}\end{array}$ & $\begin{array}{l}0.452_{-0.036}^{+0.061} \\
0.450_{-0.036}^{+0.061} \\
0.456_{-0.036}^{+0.060}\end{array}$ \\
\hline B090 & WFC/F814 & $2.005_{-0.263}^{+0.617}$ & $\begin{array}{r}\text { K66 } \\
\text { W } \\
\text { S }\end{array}$ & $\begin{array}{l}0.259 \\
0.445 \\
0.259\end{array}$ & $\begin{array}{l}-0.318_{-0.069}^{+0.113} \\
-0.132_{-0.198}^{+0.329} \\
-0.318_{-0.068}^{+0.101}\end{array}$ & $\begin{array}{l}0.636_{-0.034}^{+0.060} \\
0.639_{-0.036}^{+0.060} \\
0.608_{-0.033}^{+0.060}\end{array}$ & $\begin{array}{l}0.633_{-0.034}^{+0.060} \\
0.632_{-0.039}^{+0.060} \\
0.621_{-0.033}^{+0.060}\end{array}$ & $\begin{array}{l}0.608_{-0.035}^{+0.060} \\
0.595_{-0.047}^{+0.062} \\
0.601_{-0.035}^{+0.060}\end{array}$ & $\begin{array}{l}0.556_{-0.037}^{+0.060} \\
0.543_{-0.053}^{+0.063} \\
0.548_{-0.036}^{+0.060}\end{array}$ & $\begin{array}{l}0.542_{-0.038}^{+0.061} \\
0.524_{-0.065}^{+0.065} \\
0.537_{-0.037}^{+0.061}\end{array}$ \\
\hline
\end{tabular}

Note. - See text for column descriptions. Table 8 is available in its entirety in the electronic edition of the Journal. A short extract from it is shown here, for guidance regarding its form and content. 
Table 9. Measured Aperture Velocity Dispersions for GCs in M31

\begin{tabular}{lccc}
\hline \hline Name & $\begin{array}{c}\sigma_{\text {ap }} \\
{\left[\mathrm{km} \mathrm{s}^{-1}\right]}\end{array}$ & $\begin{array}{c}\text { Aperture } \\
{[\operatorname{arcsec}]}\end{array}$ & Source \\
\hline
\end{tabular}

Clusters in the present sample

\begin{tabular}{|c|c|c|c|}
\hline B006 & $11.56 \pm 0.11$ & 1.60 & Djorgovski et al. (1997) \\
\hline B006 & $10.6 \pm 0.4$ & 1.80 & Dubath \& Grillmair (1997) \\
\hline B012 & $16.15 \pm 0.45$ & 1.60 & Djorgovski et al. (1997) \\
\hline B020 & $14.27 \pm 0.24$ & 1.60 & Diorgovski et al. (1997) \\
\hline B020 & $15.3 \pm 0.5$ & 1.55 & Dubath \& Grillmair (1997) \\
\hline B020 & $18 \pm 3$ & 1.2 & Peterson (1989) \\
\hline B023 & $25.46 \pm 0.90$ & 1.60 & Diorgovski et al. (1997) \\
\hline B023 & $24 \pm 6$ & 1.2 & Peterson (1989) \\
\hline B037 & $19.55 \pm 3.50$ & 2.77 & Cohen (2006) \\
\hline B045 & $9.82 \pm 0.18$ & 1.60 & Diorgovski et al. (1997) \\
\hline B045 & $8.7 \pm 0.5$ & 1.80 & Dubath \& Grillmair (1997) \\
\hline B082 & $25 \pm 5$ & 1.2 & Peterson (1989) \\
\hline B147 & $11 \pm 2$ & 1.2 & Peterson (1989) \\
\hline B158 & $20.50 \pm 0.26$ & 1.60 & Diorgovski et al. (1997) \\
\hline B158 & $21.9 \pm 1.3$ & 1.55 & Dubath \& Grillmair (1997) \\
\hline B158 & $40 \pm 6$ & 1.2 & Peterson (1989) \\
\hline B225 & $25.94 \pm 0.38$ & 1.60 & Diorgovski et al. (1997) \\
\hline B225 & $26.9 \pm 0.5$ & 1.80 & Dubath \& Grillmair (1997) \\
\hline B225 & $<40$ & 1.2 & Peterson (1989) \\
\hline B240 & $11.92 \pm 0.22$ & 1.60 & Diorgovski et al. (1997) \\
\hline B289 & $8.43 \pm 0.44$ & 1.60 & Diorgovski et al. (1997) \\
\hline B343 & $9.08 \pm 0.39$ & 1.60 & Diorgovski et al. (1997) \\
\hline B343 & $10.2 \pm 1.7$ & 1.55 & Dubath \& Grillmair (1997) \\
\hline B358 & $8.11 \pm 0.36$ & 1.60 & Diorgovski et al. (1997) \\
\hline B358 & $7.1 \pm 1.8$ & 1.55 & Dubath \& Grillmair (1997) \\
\hline B373 & $12.58 \pm 0.15$ & 1.60 & Diorgovski et al. (1997) \\
\hline B379 & $8.15 \pm 0.20$ & 1.60 & Diorgovski et al. (1997) \\
\hline B384 & $10.10 \pm 0.16$ & 1.60 & Diorgovski et al. (1997) \\
\hline B384 & $9.1 \pm 0.5$ & 1.55 & Dubath \& Grillmair (1997) \\
\hline B386 & $11.49 \pm 0.24$ & 1.60 & Diorgovski et al. (1997) \\
\hline B405 & $8.57 \pm 0.45$ & 1.60 & Djorgovski et al. (1997) \\
\hline B407 & $9.52 \pm 0.11$ & 1.60 & Diorgovski et al. (1997) \\
\hline G001 & $25.06 \pm 0.32$ & 1.60 & Diorgovski et al. (1997) \\
\hline G001 & $21.47 \pm 1.60$ & 2.77 & Cohen (2006) \\
\hline G002 & $9.70 \pm 0.29$ & 1.60 & Diorgovski et al. (1997) \\
\hline
\end{tabular}

Clusters not in the present sample

$\begin{array}{rrrl}\text { B019 } & 19 \pm 3 & 1.2 & \text { Peterson (1989) } \\ \text { B163 } & 21 \pm 4 & 1.2 & \text { Peterson (1989) } \\ \text { B171 } & 18 \pm 3 & 1.2 & \text { Peterson (1989) } \\ \text { B193 } & 13.20 \pm 0.24 & 1.60 & \text { Diorgovski et al. (1997) } \\ \text { B193 } & 12 \pm 3 & 1.2 & \text { Peterson (1989) } \\ \text { B218 } & 17.62 \pm 0.24 & 1.60 & \text { Diorgovski et al. (1997) } \\ \text { B218 } & 16.3 \pm 0.8 & 1.55 & \text { Dubath \& Grillmair (1997) }\end{array}$


Table 9-Continued

\begin{tabular}{lrrl}
\hline \hline Name & $\begin{array}{c}\sigma_{\mathrm{ap}} \\
{\left[\mathrm{km} \mathrm{s}^{-1}\right]}\end{array}$ & $\begin{array}{c}\text { Aperture } \\
{[\operatorname{arcsec}]}\end{array}$ & Source \\
\hline B224 & $<10$ & 1.2 & Peterson (1989) \\
B381 & $<10$ & 1.2 & Peterson (1989) \\
\hline
\end{tabular}


Table 10. Fundamental plane fits

\begin{tabular}{lcccccccc}
\hline \hline \multirow{1}{*}{ Galaxy } & \multicolumn{2}{c}{$\log R_{h}=C+\gamma \log \left(R_{\mathrm{gc}}^{*}\right)$} & \multicolumn{2}{c}{$\log E_{b}^{*}=a(\log M)+b$} & & \\
& $C$ & $\gamma$ & $\mathrm{RMS}$ & $a$ & $b$ & $\mathrm{RMS}$ & $\delta \log E_{b}^{*}$ & $N$ \\
\hline Milky Way & $0.57 \pm 0.02$ & $0.47 \pm 0.05$ & 0.21 & $2.04 \pm 0.04$ & $39.63 \pm 0.21$ & 0.21 & $0.02 \pm 0.02$ & 85 \\
NGC 5128 & $0.58 \pm 0.02$ & $0.19 \pm 0.07$ & 0.19 & $2.09 \pm 0.04$ & $39.36 \pm 0.20$ & 0.19 & $-0.01 \pm 0.02$ & 104 \\
M31 & $0.43 \pm 0.02$ & $0.20 \pm 0.04$ & 0.17 & $1.92 \pm 0.03$ & $40.43 \pm 0.15$ & 0.17 & $0.15 \pm 0.02$ & 84 \\
M31(ACS/STIS) & $0.47 \pm 0.02$ & $0.11 \pm 0.04$ & 0.09 & $1.96 \pm 0.03$ & $40.19 \pm 0.15$ & 0.10 & $0.09 \pm 0.02$ & 33 \\
MCs+Fornax & $0.65 \pm 0.04$ & $0.41 \pm 0.10$ & 0.17 & $2.25 \pm 0.12$ & $38.48 \pm 0.65$ & 0.15 & $-0.07 \pm 0.04$ & 18 \\
\hline
\end{tabular}

